\title{
QUANTUM STOCHASTIC CONVOLUTION COCYCLES III
}

\author{
J. MARTIN LINDSAY AND ADAM G. SKALSKI
}

\begin{abstract}
Every Markov-regular quantum Lévy process on a multiplier $C^{*}$ bialgebra is shown to be equivalent to one governed by a quantum stochastic differential equation, and the generating functionals of norm-continuous convolution semigroups on a multiplier $C^{*}$-bialgebra are then completely characterised. These results are achieved by extending the theory of quantum Lévy processes on a compact quantum group, and more generally quantum stochastic convolution cocycles on a $C^{*}$-bialgebra, to locally compact quantum groups and multiplier $C^{*}$-bialgebras. Strict extension results obtained by Kustermans, together with automatic strictness properties developed here, are exploited to obtain existence and uniqueness for coalgebraic quantum stochastic differential equations in this setting. Then, working in the universal enveloping von Neumann bialgebra, we characterise the stochastic generators of Markov-regular, *-homomorphic (respectively completely positive and contractive) quantum stochastic convolution cocycles.
\end{abstract}

\section{INTRODUCTION}

Let $\mathbb{G}$ be a locally compact quantum semigroup, in other words a multiplier $C^{*}$-bialgebra. The main results of this paper are as follows: a concrete realisation of each abstract quantum Lévy process on $\mathbb{G}$ which is Markov-regular (that is, has norm-continuous expectation semigroup) as a quantum stochastic process in some Fock space (Corollary 6.2), and a characterisation of the generators of norm-continuous convolution semigroups of states on $\mathbb{G}$ (Theorem 6.3). These are achieved by the development of a general theory of quantum stochastic evolutions with tensor-independent identically distributed increments, culminating in infinitesimal characterisations of Markov-regular *-homomorphic, respectively completely positive and contractive, quantum stochastic convolution cocycles (Theorem 5.2). As a consequence of our results, a large family of examples of quantum Lévy processes on $\mathbb{G}$ is generated. These are indexed by a nondegenerate representation of $\mathbb{G}$ (as a $\mathrm{C}^{*}$-algebra) and a vector from the representing Hilbert space.

The notion of quantum Lévy process generalises that of classical Lévy process on a semigroup. It was first introduced by Accardi, Schürmann and von Waldenfels, in the purely algebraic framework of *-bialgebras ([ASW]), and was further developed by Schürmann and others ([Sch], [Fra]) who, in particular, extended it to other noncommutative forms of independence (free, boolean and monotone), still in the algebraic context. Inspired by Schürmann's reconstruction theorem, which states that every quantum Lévy process on a *-bialgebra can be equivalently realised on a symmetric Fock space, we first showed how the algebraic theory of quantum Lévy processes can be extended to the natural setting of quantum stochastic convolution cocycles $\left(\left[\mathrm{LS}_{1}\right]\right)$. These are families of linear maps $\left(l_{t}\right)_{t \geq 0}$ from a ${ }^{*}$-bialgebra $\mathcal{B}$ to operators on the symmetric Fock space $\mathcal{F}$, over a Hilbert space of the form

Permanent address of AGS. Department of Mathematics, University of Łódź, ul. Banacha 22, 90-238 Łódź, Poland.

2000 Mathematics Subject Classification. Primary 46L53, 81S25; Secondary 22A30, 47L25, 16W30.

Key words and phrases. Noncommutative probability, quantum stochastic, locally compact quantum group, $C^{*}$-bialgebra, $W^{*}$-bialgebra, stochastic cocycle, quantum Lévy process. 
$L^{2}\left(\mathbb{R}_{+} ; \mathrm{k}\right)$, satisfying the following cocycle identity with respect to the ampliated CCR flow $\left(\sigma_{t}\right)_{t \geq 0}$ :

$$
l_{s+t}=l_{s} \star\left(\sigma_{s} \circ l_{t}\right), \quad s, t \geq 0,
$$

together with regularity and adaptedness conditions. Our approach enabled us to then establish a theory of quantum Lévy processes on compact quantum groups and, more generally, quantum stochastic convolution cocycles on operator space coalgebras $\left(\left[\mathrm{LS}_{3}\right]\right)$. An extensive list of examples is included in Section 8 of that paper. The recent development of a satisfactory theory of locally compact quantum groups $([\mathrm{KuV}])$ provides the challenge which is addressed in the current work, namely to extend our analysis to the locally compact realm.

On the algebraic level the theories of quantum stochastic convolution cocycles on compact and locally compact quantum semigroups look similar, however their analytic aspects have a rather different nature. Whereas the coproduct on a compact quantum semigroup $B$ takes values in the spatial tensor product $B \otimes B$, which led us to an operator-space theoretic development of the theory and enabled us to establish the main results in the corresponding natural category of operator-space coalgebras, a noncompact locally compact quantum semigroup B is a nonunital $C^{*}$-algebra whose coproduct takes values in the multiplier algebra of $\mathrm{B} \otimes \mathrm{B}$. Consequently, $C^{*}$-algebraic methods come more to the fore, with the strict topology ([Lan] $]$, strict maps $\left(\left[\mathrm{Ku}_{1}\right]\right)$ and enveloping von Neumann algebras all playing crucial roles. The modern approach to quantum stochastics involves matrix spaces as a natural tool for combining $C^{*}$-algebraic quantum state spaces with von Neumann algebraic quantum noise $\left(\left[\mathrm{LW}_{3}\right]\right)$, and this was successfully exploited in $\left[\mathrm{LS}_{3}\right]$. In the context of the present paper, the strict topology on the initial $C^{*}$-algebra has to be harnessed to the matrix-space technology, so that both may be exploited in tandem.

The paper divides into three parts. The first part of the paper (Sections 1 and 2) is devoted to a careful analysis of relations between the strict topology on multiplier algebras and the norm-ultraweak hybrid topology on matrix spaces, automatic strictness of certain completely bounded maps, connections with the enveloping von Neumann algebra and ultraweak extension, and compatibility between extensions of maps continuous with respect to these topologies. Here also basic properties of multiplier $C^{*}$-bialgebras are described and a universal enveloping construction is given.

The second part of the paper (Sections 3,4 and 5), is devoted to developing the necessary quantum stochastic theory. Apart from its immediate applications in this work, the contents of these sections may be of wider interest to quantum stochastic analysts. Section 3 contains a brief summary of the relevant 'standard' theory; weak and strong coalgebraic quantum stochastic differential equations are treated in Section 4, where an automatic strictness result is used to establish uniqueness of weak solutions. Quantum stochastic convolution cocycles are analysed in Section 5, where Markov-regular completely positive contraction cocycles are shown to satisfy quantum stochastic differential equations, and the form of the stochastic generator is given, for these and for ${ }^{*}$-homomorphic cocycles.

In the last part (Section 6), quantum Lévy processes are defined in our setting, and are shown to be realisable as Fock space convolution cocycles when they are Markov-regular. This leads to the characterisation of the generating functionals of norm-continuous convolution semigroups of states on a locally compact quantum semigroup $B$ as functionals which have the form

$$
\gamma(b)=\langle\eta, \pi(b) \eta-\epsilon(b) \eta\rangle, \quad b \in \mathrm{B},
$$


where $\pi$ is a nondegenerate representation of $\mathrm{B}$ on a Hilbert space $\mathrm{h}$ and $\eta$ is a vector in h. Examples of Poisson-type (i.e. norm-continuous) convolution semigroups of states on a locally compact quantum semigroup are thereby readily produced, and their associated quantum Lévy processes constructed.

Our most satisfactory results are obtained in the case of Markov-regular quantum Lévy processes. A general theory of weakly continuous convolution semigroups of functionals on multiplier $C^{*}$-bialgebras is initiated in $\left[\mathrm{LS}_{4}\right]$. In that paper every such semigroup of states on a multiplier $C^{*}$-bialgebra of discrete type is shown to be automatically norm-continuous so that all the results of this paper apply directly in that case. In $\left[\mathrm{LS}_{4}\right]$ we have used Theorem 6.3 of this paper to derive a classical result on conditionally positive-definite functions on a compact group.

General notations. In this paper the multiplier algebra and universal enveloping von Neumann algebra of a $C^{*}$-algebra $A$ are denoted by $\widetilde{A}$ and $\bar{A}$ respectively. The notations are intended to emphasise the view of these objects as two forms of completion of $A$, enjoying the natural inclusions $A \subset \widetilde{A} \subset \bar{A}$. The symbols $\underline{\otimes}, \otimes$ and $\bar{\otimes}$ are used respectively for linear/algebraic, spatial $/$ minimal and ultraweak tensor products, of spaces and respecively, linear, completely bounded and ultraweakly continuous completely bounded maps. For any Hilbert space h, we have the ampliation and Hilbert space, given respectively by

$$
\iota_{\mathrm{h}}: B(\mathrm{H} ; \mathrm{K}) \rightarrow B(\mathrm{H} \otimes \mathrm{h} ; \mathrm{K} \otimes \mathrm{h}), T \mapsto T \otimes I_{\mathrm{h}}, \quad \text { and } \widehat{\mathrm{h}}:=\mathbb{C} \oplus \mathrm{h} .
$$

where context determines the Hilbert spaces $\mathrm{H}$ and $\mathrm{K}$.

\section{Strict extensions, tensor Products And $\chi$-STRUCTURE Maps}

In this section we recall some definitions and relevant facts about Hilbert $C^{*}$ modules $([\operatorname{Lan}])$, strict topologies $\left(\left[\mathrm{Ku}_{1}\right]\right)$, tensor products and $\mathrm{h}-\mathrm{k}$-matrix spaces $\left(\left[\mathrm{LW}_{3}\right]\right)$. We establish an automatic strictness result and show how strict tensor product constructions compare with h-matrix space constructions over a multiplier $C^{*}$-algebra. The section ends by recalling a central concept for quantum Lévy processes, namely that of $\chi$-structure maps.

Hilbert $C^{*}$-modules and multiplier algebras. For Hilbert $C^{*}$-modules $E$ and $F$ over a $C^{*}$-algebra $C, \mathcal{L}(E ; F)$ denotes the space of adjointable operators $E \rightarrow F$. Hilbert $C^{*}$-modules are endowed with a natural operator space structure under which $M_{n}(\mathcal{L}(E ; F))$ is identified with $\mathcal{L}\left(E^{n} ; F^{n}\right)$, where the column direct sums $E^{n}$ and $F^{n}$ are also Hilbert C-modules, and $\mathcal{L}(E ; F) \subset C B_{\mathrm{C}}(E ; F)$, the space of right C-linear completely bounded maps $E \rightarrow F([\mathrm{BlM}])$. The strict topology on $\mathcal{L}(E ; F)$ is the locally convex topology generated by the seminorms $T \mapsto\|T e\|+\left\|T^{*} f\right\|$ $(e \in E, f \in F)$; it is Hausdorff and complete. The closed subspace of $\mathcal{L}(E ; F)$ generated by the elementary maps $|f\rangle\langle e|: x \mapsto f\langle e, x\rangle(e \in E, f \in F)$ is denoted $\mathcal{K}(E ; F)$. The unit ball of $\mathcal{K}(E ; F)$ is strictly dense in that of $\mathcal{L}(E ; F), \mathcal{K}(E)$ is a $C^{*}$-algebra and $\mathcal{L}(E)$ is a model for its multiplier algebra. In particular, viewing a $C^{*}$-algebra $\mathrm{A}$ as a Hilbert $C^{*}$-module over itself, $\mathcal{K}(\mathrm{A})=\mathrm{A}$ so that $\mathcal{L}(\mathrm{A})$ is a model for the multipler algebra $\widetilde{\mathrm{A}}$. A net of positive contractions $\left(e_{i}\right)_{i \in I}$ in $\mathrm{A}$ is an approximate identity for $\mathrm{A}$ if and only if $e_{i} \rightarrow 1_{\widetilde{\mathrm{A}}}$ strictly. When $\mathrm{A}$ is unital the strict topology coincides with the norm topology. For Hilbert spaces $\mathrm{h}$ and $\mathrm{k}$, $|\mathrm{h}\rangle:=B(\mathbb{C} ; \mathrm{h})$ and $|\mathrm{k}\rangle$ are Hilbert $C^{*}$-modules over $\mathbb{C}, \mathcal{L}(|\mathrm{h}\rangle ;|\mathrm{k}\rangle)$ and $\mathcal{K}(|\mathrm{h}\rangle ;|\mathrm{k}\rangle)$ are naturally identified with $B(\mathrm{~h} ; \mathrm{k})$ and $K(\mathrm{~h} ; \mathrm{k})$ respectively, and the strict topology on $\mathcal{L}(|\mathrm{h}\rangle ;|\mathrm{k}\rangle)$ corresponds to the strong*-topology on $B(\mathrm{~h} ; \mathrm{k})$. When a $C^{*}$-algebra

$A$ acts nondegenerately on a Hilbert space $h$, the multiplier algebra $\widetilde{A}$ is realised as the double centraliser of $\mathrm{A}$ in $B(\mathrm{~h}):\left\{x \in B(\mathrm{~h}): \forall_{a \in \mathrm{A}} x a, a x \in \mathrm{A}\right\}$, the inclusion 
$\widetilde{A} \subset A^{\prime \prime}$ holds and bounded strictly convergent nets in $\widetilde{A}$ converge (strong*- and thus) $\sigma$-weakly.

Two elementary classes of strictly continuous maps that feature below are component maps $\varepsilon_{k l}: \mathcal{L}\left(E_{1} \oplus E_{2}\right) \rightarrow \mathcal{L}\left(E_{k} ; E_{l}\right), T=\left[T_{i j}\right] \mapsto T_{k l}$, where the column direct sum $E_{1} \oplus E_{2}$ is a Hilbert C-module, and multiplication operators $\mathcal{L}(E ; F) \rightarrow \mathcal{L}\left(E^{\prime} ; F^{\prime}\right), T \mapsto X^{*} T Y$, where $X \in \mathcal{L}\left(F^{\prime} ; F\right)$ and $Y \in \mathcal{L}\left(E^{\prime} ; E\right)$ for Hilbert C-modules $E^{\prime}$ and $F^{\prime}$.

Strict maps and extensions. There is a more prevalent notion in the theory than strict continuity. A bounded operator $\varphi$ from $\mathcal{K}=\mathcal{K}(E ; F)$ to $\mathcal{L}^{\prime}=\mathcal{L}\left(E^{\prime} ; F^{\prime}\right)$ where $E^{\prime}$ and $F^{\prime}$ are Hilbert $C^{*}$-modules over a $C^{*}$-algebra $C^{\prime}$, is called strict if it is strictly continuous on bounded sets; the collection of such maps, denoted $B_{\beta}\left(\mathcal{K} ; \mathcal{L}^{\prime}\right)$, is a closed subspace of $B\left(\mathcal{K} ; \mathcal{L}^{\prime}\right)$; we describe some of its contents below. Here we are particularly interested in the classes $B_{\beta}(\mathrm{A} ; B)$ for $C^{*}$-algebras $\mathrm{A}$ and spaces $B$ of the form $B(\mathrm{~h} ; \mathrm{k})$ where $\mathrm{h}$ and $\mathrm{k}$ are Hilbert spaces. An important general class of strict maps is the set of ${ }^{*}$-homomorphisms $\varphi: \mathrm{A} \rightarrow \mathcal{L}(E)$, for a $C^{*}$-algebra $\mathrm{A}$ and Hilbert $C^{*}$-module $E$, which are nondegenerate in the sense that $\overline{\operatorname{Lin}} \varphi(\mathrm{A}) E=E$.

For a $C^{*}$-algebra $A$, let $\bar{A}$ denote its universal enveloping von Neumann algebra, let $\rho$ be the embedding $\mathrm{A} \rightarrow \overline{\mathrm{A}}$ and let $\iota$ be the inclusion/natural map $\overline{\mathrm{A}}_{*} \rightarrow$ $\overline{\mathrm{A}}^{*}=\left(\overline{\mathrm{A}}_{*}\right)^{* *}$. The map $\iota^{*} \circ \rho^{* *}: \mathrm{A}^{* *} \rightarrow\left(\overline{\mathrm{A}}_{*}\right)^{*}=\overline{\mathrm{A}}$ is a ${ }^{*}$-isomorphism for the common Arens product on $\mathrm{A}^{* *}$ and a weak ${ }^{*}-\sigma$-weak homeomorphism. Since $\mathrm{A}$ acts nondegenerately in the universal representation, $\widetilde{A}$ may be viewed as a subalgebra of $\bar{A}$. All of this is well-known. For ease of reference we collect together some extension properties which will play an important role here. The notation $B_{\sigma}$ stands for bounded ultraweakly continuous.

Theorem 1.1. Let $\mathrm{A}$ be a $C^{*}$-algebra with multiplier algebra $\widetilde{\mathrm{A}}$ and universal enveloping von Neumann algebra $\overline{\mathrm{A}}$.

(a) Let $\varphi \in B_{\beta}(\mathrm{A} ; \mathcal{L})$ where $\mathcal{L}=\mathcal{L}(E ; F)$ for $C^{*}$-modules $E$ and $F$ over a $C^{*}$ algebra $\mathrm{C}$. Then $\varphi$ has a unique strict extension $\widetilde{\varphi}: \widetilde{\mathrm{A}} \rightarrow \mathcal{L}$, moreover $\widetilde{\varphi}$ is bounded and $\|\widetilde{\varphi}\|=\|\varphi\|$.

(b) Let $\psi \in B(\mathrm{~A} ; B)$ where $B=B(\mathrm{~h} ; \mathrm{k})$ for Hilbert spaces $\mathrm{h}$ and $\mathrm{k}$. Then $\psi$ has a unique normal extension $\bar{\psi} \in B_{\sigma}(\overline{\mathrm{A}} ; B)$, moreover $\|\bar{\psi}\|=\|\psi\|$.

(c) Let $\phi \in B_{\beta}(\mathrm{A} ; B)$ where $B=B(\mathrm{~h} ; \mathrm{k})=\mathcal{L}(|\mathrm{h}\rangle ;|\mathrm{k}\rangle)$ for Hilbert spaces $\mathrm{h}$ and k. Then $\widetilde{\phi}=\left.\bar{\phi}\right|_{\tilde{A}}$.

In $(a), \widetilde{\varphi^{\dagger}}=\widetilde{\varphi}^{\dagger}$ where $\varphi^{\dagger}: \mathrm{A} \rightarrow \mathcal{L}(F ; E)$ is defined by $\varphi^{\dagger}\left(a^{*}\right)=\varphi(a)^{*}$; similarly, in $(b) \overline{\psi^{\dagger}}=\bar{\psi}^{\dagger}$. When $F=E, \widetilde{\varphi}$ is positive/completely positive/multiplicative if $\varphi$ is, and likewise for $\psi$ and $\bar{\psi}$ when $\mathrm{k}=\mathrm{h}$.

Proof. (a) is proved in $\left[\mathrm{Ku}_{1}\right]$ in the case $E=F=\mathrm{C}$. The general case is obtained by applying this case with $\mathrm{C}=\mathcal{K}(E \oplus F)$ and composing with the strict map $\mathcal{L}(E \oplus F) \rightarrow \mathcal{L}(E ; F), T=\left[T_{i, j}\right] \mapsto T_{21}$. (b) is well-known: set $\bar{\psi}:=\iota^{*} \circ \psi^{* *} \circ j$ where $\iota$ is the natural map/inclusion $B_{*} \rightarrow\left(B_{*}\right)^{* *}=B^{*}$ and $j$ is the natural isometric isomorphism $\bar{A} \rightarrow A^{* *}$. Since the unit ball of $A$ is strictly dense in that of $\widetilde{A}$ ([Lan], Proposition 1.4), (c) follows from the fact that strictly convergent bounded nets converge $\sigma$-weakly. The last part follows from Kaplansky's density theorem and its Hilbert $C^{*}$-module counterpart (just used), and the separate strict (respectively $\sigma$-weak) continuity of multiplication and corresponding continuity of the adjoint operation, in a multiplier algebra (respectively von Neumann algebra). 
Remarks. (i) The extensions commute with matrix liftings:

$$
\begin{aligned}
& \varphi^{(n) \sim}=\widetilde{\varphi}^{(n)}: M_{n}(\mathrm{~A})^{\sim}=M_{n}(\widetilde{\mathrm{A}}) \rightarrow M_{n}(\mathcal{L})=\mathcal{L}\left(E^{n} ; F^{n}\right) \\
& \overline{\psi^{(n)}}=\bar{\psi}^{(n)}: \overline{M_{n}(\mathrm{~A})}=M_{n}(\overline{\mathrm{A}}) \rightarrow M_{n}(B)=B\left(\mathrm{~h}^{n} ; \mathrm{k}^{n}\right),
\end{aligned}
$$

so $\|\widetilde{\varphi}\|_{\mathrm{cb}}=\|\varphi\|_{\mathrm{cb}}$ when $\varphi \in C B_{\beta}(\mathrm{A} ; \mathcal{L})$ and $\|\bar{\phi}\|_{\mathrm{cb}}=\|\phi\|_{\mathrm{cb}}$ when $\psi \in C B(\mathrm{~A} ; B)$.

(ii) Clearly the range of $\widetilde{\varphi}$ is contained in the strict closure of the range of $\varphi$, and the range of $\bar{\psi}$ is contained in the $\sigma$-weak closure of the range of $\psi$.

(iii) As a consequence of (a), strict maps may be composed in the following sense: if $\varphi_{1} \in B_{\beta}\left(\mathrm{A}_{1} ; \widetilde{\mathrm{A}}_{2}\right)$ and $\varphi_{2} \in B_{\beta}\left(\mathrm{A}_{2} ; \widetilde{\mathrm{A}}_{3}\right)$, for $C^{*}$-algebras $\mathrm{A}_{1}, \mathrm{~A}_{2}$ and $\mathrm{A}_{3}$, then $a \mapsto$ $\widetilde{\varphi}_{2}\left(\varphi_{1}(a)\right)$ is strict with unique strict extension $\widetilde{\varphi}_{2} \circ \widetilde{\varphi}_{1}$; following the widely adopted convention (e.g. [Lan]), it is simply denoted $\varphi_{2} \circ \varphi_{1}$, thus $\varphi_{2} \circ \varphi_{1} \in B_{\beta}\left(\mathrm{A}_{1} ; \widetilde{A}_{3}\right)$. For a nondegenerate ${ }^{*}$-homomorphism $\varphi: A \rightarrow \widetilde{C}, \widetilde{\varphi}$ is a unital *-homomorphism, and conversely every nondegenerate ${ }^{*}$-homomorphism $\varphi: A \rightarrow \widetilde{C}$ is the restriction of a strict unital *-homomorphism $\widetilde{\mathrm{A}} \rightarrow \widetilde{\mathrm{C}}$.

Warning. We now write $B_{\beta}(\widetilde{\mathrm{A}} ; \mathcal{L})$ for the class of strict maps $\widetilde{\mathrm{A}} \rightarrow \mathcal{L}$ where, for us, $\mathcal{L}$ will always be either of the form $B(\mathrm{~h} ; \mathrm{k})=\mathcal{L}(|\mathrm{h}\rangle ;|\mathrm{k}\rangle)$ for Hilbert spaces $\mathrm{h}$ and $\mathrm{k}$ or of the form $\widetilde{\mathrm{C}}$ for a $C^{*}$-algebra $\mathrm{C}\left(\right.$ or both: $\left.B(\mathrm{~h})=\mathcal{L}(|\mathrm{h}\rangle)=K(\mathrm{~h})^{\sim}\right)$. Use of this notation therefore always needs to reflect the algebras of which the source (and target) multiplier algebras are.

We note that the theorem delivers a commutative diagram of isometric isomorphisms:

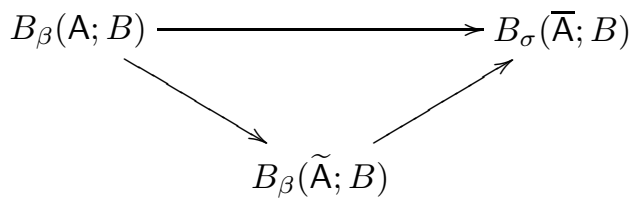

for any $C^{*}$-algebra $A$ and space $B$ of the form $B(\mathrm{~h} ; \mathrm{k})$ for Hilbert spaces $\mathrm{h}$ and $\mathrm{k}$.

Definition. Let $\varphi \in B_{\beta}(\mathrm{A} ; \mathcal{L}(E))$ for a $C^{*}$-algebra $\mathrm{A}$ and Hilbert $C^{*}$-module $E$. We call $\varphi$ preunital if its strict extension is unital: $\widetilde{\varphi}(1)=I$.

Remark. For *-homomorphisms this is equivalent to nondegeneracy; in general it is equivalent to $\varphi\left(e_{\lambda}\right) \rightarrow 1$ for some/every $C^{*}$-approximate identity $\left(e_{i}\right)_{i \in I}$ for A, but is stronger than the condition $\overline{\operatorname{Lin}} \varphi(\mathrm{A}) E=E$ ([Lan], Proposition 2.5; Corollary $5.7)$.

Automatic strictness and strict tensor products. In the next theorem we establish an automatic strictness property and identify a natural class of maps for strict tensoring. First some notation. When $A$ is the spatial tensor product $A_{1} \otimes A_{2}$, for $C^{*}$-algebras $A_{1}$ and $A_{2}, \widetilde{A}$ is denoted $A_{1} \widetilde{\otimes} A_{2}$. Note the relation

$$
\widetilde{A}_{1} \otimes \widetilde{A}_{2} \subset A_{1} \widetilde{\otimes} A_{2}
$$

Theorem 1.2. Let $\mathrm{A}, \mathrm{A}_{1}$ and $\mathrm{A}_{2}$ be $C^{*}$-algebras.

(a) Let $\varphi \in C B(\mathrm{~A} ; B)$ where $B=B(\mathrm{~h} ; \mathrm{k})$ for a Hilbert spaces $\mathrm{h}$ and $\mathrm{k}$. Then $\varphi$ is strict and $\tilde{\varphi}=\left.\bar{\varphi}\right|_{\tilde{\mathrm{A}}}$. In particular, all ${ }^{*}$-homomorphisms $\mathrm{A} \rightarrow B(\mathrm{~h})$ are strict.

(b) Let $\varphi_{i} \in \operatorname{Lin} C P_{\beta}\left(\mathrm{A}_{i} ; \widetilde{\mathrm{C}}_{i}\right)$ for $C^{*}$-algebras $\mathrm{C}_{1}$ and $\mathrm{C}_{2}$. Then, there is a unique map $\varphi_{1} \otimes \varphi_{2} \in \operatorname{Lin} C P_{\beta}\left(\mathrm{A}_{1} \otimes \mathrm{A}_{2} ; \mathrm{C}_{1} \otimes \mathrm{C}_{2}\right)$ extending the algebraic tensor product map $\varphi_{1} \underline{\otimes} \varphi_{2}$.

Proof. (a) In view of Theorem 1.1 it suffices to prove that $\varphi$ is strict. It follows from the Wittstock-Paulsen-Haagerup Decomposition Theorem ([EfR], Theorem 5.3.3) 
that $\varphi=\psi \circ \pi$ where $\pi$ is a *homomorphism $\mathrm{A} \rightarrow B(\mathrm{H})$ for some Hilbert space $\mathrm{H}$ and $\psi: B(\mathrm{H}) \rightarrow B$ is of the form $X \mapsto R^{*} X S$, for some operators $R \in B(\mathrm{k} ; \mathrm{H})$ and

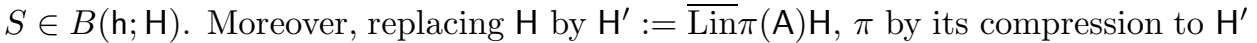
$R$ and $S$ by $R P$ and $S P$ where $P$ is the orthogonal projection $\mathrm{H} \rightarrow \mathrm{H}^{\prime}$, if necessary, we may suppose that $\pi$ is nondegenerate and therefore strict, when viewed as a map into $\mathcal{L}(|\mathrm{H}\rangle)$. Since $\psi$ is strict $\mathcal{L}(|\mathrm{H}\rangle) \rightarrow \mathcal{L}(|\mathrm{h}\rangle ;|\mathrm{k}\rangle), \varphi$ is too. (b) By linearity we may suppose that $\varphi_{1}$ and $\varphi_{2}$ are completely positive. The result then follows easily from Kasparov's extension of the Stinespring Decomposition Theorem ([Lan], Theorem $5.6)$.

Remarks. It follows from Part (a) and the remarks after Theorem 1.1 that (1.1) restricts to a commutative diagram of completely isometric isomorphisms

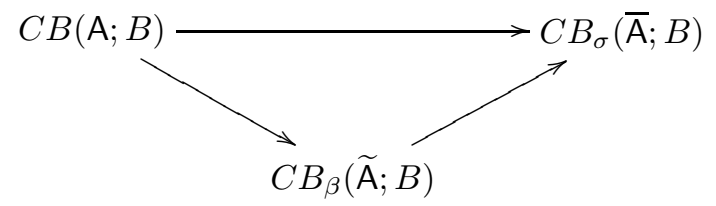

where $A$ and $B$ are as in (1.1). In particular, we have complete isometries

$$
\mathrm{A}^{*} \cong \widetilde{\mathrm{A}}_{\beta}^{*} \cong \overline{\mathrm{A}}_{*} \text {. }
$$

By operator space considerations $\operatorname{Ran}\left(\varphi_{1} \otimes \varphi_{2}\right) \subset \widetilde{C}_{1} \otimes \widetilde{C}_{2}$. Part (b) leads to the following useful notation. For $\varphi_{i} \in \operatorname{Lin} C P_{\beta}\left(\mathrm{A}_{i} ; \widetilde{C}_{i}\right)(i=1,2)$ we denote the unique strict extension of $\varphi_{1} \otimes \varphi_{2}$ by $\varphi_{1} \widetilde{\otimes} \varphi_{2}$. Thus

$$
\varphi_{1} \widetilde{\otimes} \varphi_{2} \in \operatorname{Lin} C P_{\beta}\left(\mathrm{A}_{1} \widetilde{\otimes} \mathrm{A}_{2} ; \mathrm{C}_{1} \widetilde{\otimes} \mathrm{C}_{2}\right) \text {. }
$$

Note the following consequence of Part (a) and its proof, which provides a source for tensoring as in Part (b).

Corollary 1.3. For a $C^{*}$-algebra $\mathrm{A}$ and Hilbert space $\mathrm{h}$,

$$
C B(\mathrm{~A} ; B(\mathrm{~h}))=\operatorname{Lin} C P_{\beta}(\mathrm{A} ; B(\mathrm{~h})) .
$$

Remark. This 'strict decomposability' property is very useful. For a general multiplier algebra target space, completely bounded maps need neither be strict nor be linear combinations of completely positive maps.

h-k-Matrix spaces. Let $\mathrm{V}$ be an operator space in $B(\mathrm{H} ; \mathrm{K})$, set $B=B(\mathrm{~h} ; \mathrm{k})$ for two further Hilbert spaces $\mathrm{h}$ and $\mathrm{k}$ with total subsets $S$ and $T$, and let $Z \in$ $B(\mathrm{H} \otimes \mathrm{h} ; \mathrm{K} \otimes \mathrm{k})=B(\mathrm{H} ; \mathrm{K}) \bar{\otimes}$. Then the following are equivalent:

$$
\begin{aligned}
& E^{\xi} Z E_{\eta} \in \mathrm{V} \text { for all } \xi \in T, \eta \in S \\
& \left.\operatorname{id}_{B(\mathrm{H} ; \mathrm{K})} \bar{\otimes} \omega\right)(Z) \in \mathrm{V} \text { for all } \omega \in B_{*}
\end{aligned}
$$

where, for a Hilbert space vector $\xi$,

$$
E_{\xi}:=I \otimes|\xi\rangle: u \mapsto u \otimes \xi \text { and } E^{\xi}:=\left(E_{\xi}\right)^{*}=I \otimes\langle\xi|,
$$

and $I$ is the identity operator on the appropriate Hilbert space. The collection of operators $Z$ enjoying this property is an operator space which is denoted $\mathrm{V} \otimes_{\mathrm{M}} B$ and called the (right) h-k-matrix space over $\mathrm{V}$. It is situated between the norm-spatial and ultraweak-spatial tensor products:

$$
\mathrm{V} \otimes B \subset \mathrm{V} \otimes_{\mathrm{M}} B \subset \overline{\mathrm{V}} \bar{\otimes} B
$$

and the latter inclusion is an equality if and only if $\mathrm{V}$ is $\sigma$-weakly closed. If $\varphi \in$ $C B\left(\mathrm{~V} ; \mathrm{V}^{\prime}\right)$ for another concrete operator space $\mathrm{V}^{\prime}$ then there is a unique map, its $\mathrm{h}$ k-lifting, denoted $\varphi \otimes_{\mathrm{M}} \mathrm{id}_{B}$, from $\mathrm{V} \otimes_{\mathrm{M}} B$ to $\mathrm{V}^{\prime} \otimes_{\mathrm{M}} B$ satisfying $E^{\xi}\left(\varphi \otimes_{\mathrm{M}} \operatorname{id}_{B}\right)(Z) E_{\eta}=$ $\varphi\left(E^{\xi} Z E_{\eta}\right)$ for all $\xi \in \mathrm{k}, \eta \in \mathrm{h}$ and $Z \in \mathrm{V} \otimes_{\mathrm{M}} B$; it is completely bounded, with 
$\left\|\varphi \otimes_{\mathrm{M}} \operatorname{id}_{B}\right\|_{\mathrm{cb}}=\|\varphi\|_{\mathrm{cb}}$ and completely isometric if $\varphi$ is (unless $B=\{0\}$ ), moreover it extends $\varphi \otimes \operatorname{id}_{B}$, and it coincides with $\varphi \bar{\otimes} \operatorname{id}_{B}$ when $\mathrm{V}$ is $\sigma$-weakly closed and $\varphi$ is $\sigma$-weakly continuous. The next proposition confirms the compatibility of h-k-matrix spaces and h-k-liftings on the one hand, and strict tensor products of algebras and strict maps on the other. First note the identity

$$
\|\eta\|^{2} R E_{\eta} X=R(X \otimes|\eta\rangle\langle\eta|) E_{\eta},
$$

for Hilbert space operators $R \in B(\mathrm{H} \otimes \mathrm{h} ; \mathrm{H})$ and $X \in B(\mathrm{H})$ and vectors $\eta \in \mathrm{h}$.

Proposition 1.4. Let $\mathrm{A}$ be a $C^{*}$-algebra and let $\mathrm{h}$ be a Hilbert space. Then, in any faithful nondegenerate representation of $\mathrm{A}$ (such as its universal representation),

$$
\mathrm{A} \widetilde{\otimes} K(\mathrm{~h}) \subset \widetilde{\mathrm{A}} \otimes_{\mathrm{M}} B(\mathrm{~h}),
$$

for the induced concrete realisations of $\widetilde{\mathrm{A}}$ and $\mathrm{A} \widetilde{\otimes} K(\mathrm{~h})$. Moreover, if $\psi \in \operatorname{Lin} C P_{\beta}(\mathrm{A} ; \widetilde{\mathrm{C}})$ for another $C^{*}$-algebra $\mathrm{C}$, also faithfully and nondegenerately represented, then

$$
\psi \widetilde{\otimes} \operatorname{id}_{K(\mathrm{~h})} \subset \widetilde{\psi} \otimes_{\mathrm{M}} \operatorname{id}_{B(\mathrm{~h})} .
$$

Proof. Set $B=B(\mathrm{~h})$ and $K=K(\mathrm{~h})$. Let $T \in \mathrm{A} \widetilde{\otimes} K$ and $\zeta, \eta \in \mathrm{h}$. First note that (1.8) implies that

$$
\|\eta\|^{2} E^{\zeta} T E_{\eta} a=E^{\zeta} T(a \otimes|\eta\rangle\langle\eta|) E_{\eta}, \quad a \in \mathrm{A} ;
$$

similarly,

$$
\|\zeta\|^{2} a E^{\zeta} T E_{\eta}=E^{\zeta}(a \otimes|\zeta\rangle\langle\zeta|) T E_{\eta}, \quad a \in \mathrm{A} ;
$$

and so $E^{\zeta} T E_{\eta} \in \widetilde{\mathrm{A}}$. Thus $T \in \widetilde{\mathrm{A}} \otimes_{\mathrm{M}} B$. This proves that $\mathrm{A} \widetilde{\otimes} K \subset \widetilde{\mathrm{A}} \otimes_{\mathrm{M}} B$, and (1.9) and (1.9) now imply that the map

$$
T \in \mathrm{A} \widetilde{\otimes} K \mapsto E^{\zeta} T E_{\eta} \in \widetilde{\mathrm{A}}
$$

is strictly continuous. Therefore the maps

$$
E^{\zeta}\left(\widetilde{\psi} \otimes_{\mathrm{M}} \operatorname{id}_{B}\right)(\cdot) E_{\eta}=\widetilde{\psi}\left(E^{\zeta} \cdot E_{\eta}\right) \text { and } E^{\zeta}\left(\psi \widetilde{\otimes} \operatorname{id}_{K}\right)(\cdot) E_{\eta}
$$

are strictly continuous $\mathrm{A} \widetilde{\otimes} K \rightarrow \widetilde{\mathrm{C}}$ and agree on the strictly dense subspace $\mathrm{A} \otimes K$. They therefore agree on $\mathrm{A} \widetilde{\otimes} K$ and the result follows.

Remark. In the universal representation of $\mathrm{A}$ we have the further compatibility relations,

$$
\widetilde{\mathrm{A}} \otimes_{\mathrm{M}} B(\mathrm{~h}) \subset \overline{\mathrm{A}} \bar{\otimes} B(\mathrm{~h}) \text { and } \widetilde{\psi} \otimes_{\mathrm{M}} \mathrm{id}_{B(\mathrm{~h})} \subset \bar{\psi} \bar{\otimes} \mathrm{id}_{B(\mathrm{~h})} .
$$

$C^{*}$-algebras with character. The following notion plays an important role in the theory. Recall the notation (0.1).

Definition. A $\chi$-structure map on a $C^{*}$-algebra with character $(\mathrm{A}, \chi)$ is a linear map $\varphi: A \rightarrow B(\widehat{h})$, for some Hilbert space $\mathrm{h}$, satisfying

$$
\varphi\left(a^{*} b\right)=\varphi(a)^{*} \chi(b)+\chi(a)^{*} \varphi(b)+\varphi(a)^{*} \Delta \varphi(b),
$$

where $\Delta:=\left[\begin{array}{ll}{ }^{0} & \\ { }^{\mathrm{h}}\end{array}\right] \in B(\widehat{\mathrm{h}})$ (no relation to coproducts).

In terms of its block matrix form $\varphi=\left[\begin{array}{ll}\gamma & \mu \\ \lambda & \nu\end{array}\right], \lambda$ is a kind of derivation (see below) and $\mu=\lambda^{\dagger}: a \mapsto \lambda\left(a^{*}\right)^{*}$. More specifically, the following result, established in $\left[\mathrm{LS}_{3}\right]$, gives the general form of $\chi$-structure maps.

Theorem 1.5. Let $(\mathrm{A}, \chi)$ be a $C^{*}$-algebra with character and let $\varphi$ be a linear map $\mathrm{A} \rightarrow B(\widehat{\mathrm{h}})$, for some Hilbert space $\mathrm{h}$. Then the following are equivalent.

(i) $\varphi$ is a $\chi$-structure map. 
(ii) $\varphi$ has block matrix form

$a \mapsto\left[\begin{array}{cc}\gamma(a) & \langle\xi| \nu(a) \\ \nu(a)|\xi\rangle & \nu(a)\end{array}\right]$ where $\gamma:=\omega_{\xi} \circ \nu$ for $\nu:=\pi-\iota_{\mathrm{h}} \circ \chi$,

in which $(\pi, \mathrm{h})$ is a representation of $\mathrm{A}$ (as a $C^{*}$-algebra) and $\xi$ is a vector in $\mathrm{h}$.

If $\varphi$ is a $\chi$-structure map with such a block matrix form then it is necessarily strict, moreover $\pi$ is nondegenerate if and only if $\widetilde{\varphi}(1)=0$.

Proof. The first part is Theorem A6 of $\left[\mathrm{LS}_{3}\right]$. This implies that $\varphi$ is completely bounded and so, by Theorem 1.2, $\varphi$ is strict. After strict extension, the last part now follows by inspection.

We say that the $\chi$-structure map $\varphi$ is implemented by the pair $(\pi, \xi)$. Note the following alternative expression:

$$
\varphi(a)=\left[\begin{array}{c}
\langle\xi| \\
I_{\mathrm{h}}
\end{array}\right]\left(\pi(a)-\chi(a) I_{\mathrm{h}}\right)\left[|\xi\rangle \quad I_{\mathrm{h}}\right] \quad(a \in \mathrm{A})
$$

Remarks. Thus $\lambda$ is a $\pi$ - $\chi$-derivation, in other words $\lambda(a b)=\lambda(a) \chi(b)+\pi(a) \lambda(b)$ $(a, b \in \mathrm{A})$, which is implemented.

By the separate strict $/ \sigma$-weak continuity of multiplication, it follows that if $\varphi$ is a $\chi$-structure map then $\widetilde{\varphi}$ is a $\widetilde{\chi}$-structure map and $\bar{\varphi}$ is a $\bar{\chi}$-structure map.

We shall need the following result in Section 6 .

Lemma 1.6. Let $(\mathrm{A}, \chi)$ be a $C^{*}$-algebra with character. Then, for any functional $\gamma \in \mathrm{A}^{*}$, if $\gamma$ is positive on $\operatorname{Ker} \chi$ then $\widetilde{\gamma}$ is positive on $\operatorname{Ker} \tilde{\chi}$ and $\bar{\gamma}$ is positive on Ker $\bar{\chi}$.

Proof. It suffices to prove that $\mathrm{A}_{+} \cap \operatorname{Ker} \chi$ is strictly dense in $\widetilde{\mathrm{A}}_{+} \cap \operatorname{Ker} \tilde{\chi}$ and $\sigma$ weakly dense in $\overline{\mathrm{A}}_{+} \cap \operatorname{Ker} \bar{\chi}$. Let $a \in \widetilde{\mathrm{A}}_{+} \cap \operatorname{Ker} \tilde{\chi}$. The Kaplansky Density Theorem for multiplier algebras ([Lan], Proposition 1.4) implies that there is a bounded net $\left(c_{i}\right)_{i \in I}$ of selfadjoint elements in A converging strictly to $a^{1 / 4}$. Set $a_{i}=b_{i}^{*} b_{i}$ where

$$
b_{i}:=c_{i}\left(c_{i}-\chi\left(c_{i}\right)\right) \in \operatorname{Ker} \chi .
$$

Then $a_{i} \in \mathrm{A}_{+} \cap \operatorname{Ker} \chi$ and separate strict continuity of multiplication on bounded subsets of $\mathrm{A}$, and strictness of $\chi$, imply that $\left(a_{i}\right)_{i \in I}$ converges strictly to $a$. The ultraweak density of $A_{+} \cap \operatorname{Ker} \chi$ in $\bar{A}_{+} \cap \operatorname{Ker} \bar{\chi}$ is proved similarly, by appealing to the standard Kaplansky Density Theorem (for von Neumann algebras).

\section{Multiplier $C^{*}$-Bialgebras}

It is convenient to consider bialgebras in both the $C^{*}$ - and $W^{*}$ - categories and a universal enveloping operation linking the two.

Definition. A (multiplier) $C^{*}$-bialgebra is a $C^{*}$-algebra B with coproduct, that is a nondegenerate ${ }^{*}$-homomorphism $\Delta: \mathrm{B} \rightarrow \mathrm{B} \widetilde{\otimes} \mathrm{B}$ satisfying the coassociativity conditions

$$
\left(\mathrm{id}_{\mathrm{B}} \otimes \Delta\right) \circ \Delta=\left(\Delta \otimes \mathrm{id}_{\mathrm{B}}\right) \circ \Delta .
$$

A counit for $(\mathrm{B}, \Delta)$ is a character $\epsilon$ on $\mathrm{B}$ satisfying the counital property:

$$
\left(\operatorname{id}_{\mathrm{B}} \otimes \epsilon\right) \circ \Delta=\left(\epsilon \otimes \operatorname{id}_{\mathrm{B}}\right) \circ \Delta=\mathrm{id}_{\mathrm{B}} .
$$

Remarks. The above definitions extend those for unital $C^{*}$-bialgebras, for which $\widetilde{B}=\mathrm{B}$ and $\mathrm{B} \widetilde{\otimes} \mathrm{B}=\mathrm{B} \otimes \mathrm{B}$. The strict extension of a coproduct is a unital $*_{-}$ homomorphism and the strict extension of a counit is a character on $\widetilde{B}$. Note however that, in general, $(\widetilde{\mathrm{B}}, \widetilde{\Delta})$ is not itself a $C^{*}$-bialgebra as the inclusion $\widetilde{\mathrm{B}} \otimes \widetilde{\mathrm{B}} \subset$ $\mathrm{B} \widetilde{\otimes} \mathrm{B}$ is usually proper. 
Examples of counital $C^{*}$-bialgebras include locally compact quantum groups in the universal setting $\left(\left[\mathrm{Ku}_{2}\right]\right)$, in particular all coamenable locally compact quantum groups are included. If the assumptions on the coproduct $\Delta$ are weakened to it being completely positive, strict and preunital then the resulting structure is called a (multiplier) $C^{*}$-hyperbialgebra (cf. $\left.[\mathrm{ChV}]\right)$.

Let $\mathrm{B}$ be a $C^{*}$-bialgebra. The convolute of $\phi_{1} \in \operatorname{Lin} C P_{\beta}\left(\mathrm{B} ; \widetilde{\mathrm{A}}_{1}\right)$ and $\phi_{2} \in$ $\operatorname{Lin} C P_{\beta}\left(\mathrm{B} ; \widetilde{\mathrm{A}}_{2}\right)$ for $C^{*}$-algebras $\mathrm{A}_{1}$ and $\mathrm{A}_{2}$ is defined by

$$
\phi_{1} \star \phi_{2}:=\left(\phi_{1} \otimes \phi_{2}\right) \circ \Delta \in \operatorname{Lin} C P_{\beta}\left(\mathrm{B} ; \mathrm{A}_{1} \widetilde{\otimes} \mathrm{A}_{2}\right) .
$$

We denote its strict extension by $\phi_{1} \widetilde{\star} \phi_{2}$. Associativity of both of these convolutions follows from the associativity of $\widetilde{\otimes}$ and coassociativity of $\widetilde{\Delta}$. For each $C^{*}$-algebra A define a map

$$
R_{\mathrm{A}}: \operatorname{Lin} C P_{\beta}(\mathrm{B} ; \widetilde{\mathrm{A}}) \rightarrow C B_{\beta}(\mathrm{B} ; \mathrm{B} \widetilde{\otimes} \mathrm{A}), \quad \phi \rightarrow \mathrm{id}_{\mathrm{B}} \star \phi=\left(\mathrm{id}_{\mathrm{B}} \otimes \phi\right) \circ \Delta .
$$

In case $\mathrm{A}=\mathbb{C}, \operatorname{Lin} C P_{\beta}(\mathrm{B} ; \widetilde{\mathrm{A}})$ is simply $\mathrm{B}^{*}$ and we have

$$
R_{\mathbb{C}}\left(\varphi_{1} \star \varphi_{2}\right)=R_{\mathbb{C}} \varphi_{1} \circ R_{\mathbb{C}} \varphi_{2}, \quad \varphi_{1}, \varphi_{2} \in \mathrm{B}^{*} .
$$

When $\mathrm{B}$ is counital each $R_{\mathrm{A}}$ has left-inverse

$$
E_{\mathrm{A}}: C B_{\beta}(\mathrm{B} ; \mathrm{B} \widetilde{\otimes} \mathrm{A}) \rightarrow C B_{\beta}(\mathrm{B} ; \widetilde{\mathrm{A}}), \quad \psi \rightarrow\left(\epsilon \otimes \mathrm{id}_{\mathrm{B}}\right) \circ \psi .
$$

Remarks. By the complete positivity and strictness of the coproduct

$$
R_{\mathrm{A}}\left(C P_{\beta}(\mathrm{B} ; \widetilde{\mathrm{A}})\right) \subset C P_{\beta}(\mathrm{B} ; \mathrm{B} \widetilde{\otimes} \mathrm{A})
$$

for any $C^{*}$-algebra $A$. In particular, by (1.6),

$$
R_{K(\mathrm{~h})}(C B(\mathrm{~B} ; B(\mathrm{~h}))) \subset \operatorname{Lin} C P_{\beta}(\mathrm{B} ; \mathrm{B} \widetilde{\otimes} K(\mathrm{~h})) .
$$

Note also that, when $\varphi_{1} \in C B\left(\mathrm{~B} ; B\left(\mathrm{~h}_{1}\right)\right)$ and $\varphi_{2} \in C B\left(\mathrm{~B} ; B\left(\mathrm{~h}_{2}\right)\right)$ for Hilbert spaces $\mathrm{h}_{1}$ and $\mathrm{h}_{2}$,

$$
\varphi_{1} \star \varphi_{2} \in C B\left(\mathrm{~B} ; B\left(\mathrm{~h}_{1} \otimes \mathrm{h}_{2}\right)\right) .
$$

For convenience we summarise useful properties of the $\mathcal{R}$-maps next.

Proposition 2.1. Let $\mathrm{B}$ be a $C^{*}$-bialgebra and let $\mathrm{A}$ be a $C^{*}$-algebra. Then $R_{\mathrm{A}}$ is a completely contractive map into $C B_{\beta}(\mathrm{B} ; \mathrm{B} \widetilde{\otimes} \mathrm{A})$ with image in the subspace Lin $C P_{\beta}(\mathrm{B} ; \mathrm{B} \widetilde{\otimes} \mathrm{A})$ and, after strict extension, $R_{\mathbb{C}}$ is furthermore a homomorphism of Banach algebras: $\left(\mathrm{B}^{*}, \star\right) \cong\left((\widetilde{\mathrm{B}})_{\beta}^{*}, \widetilde{\star}\right) \rightarrow C B_{\beta}(\widetilde{\mathrm{B}})$. When $\mathrm{B}$ is counital, $R_{\mathrm{A}}$ is completely isometric with completely contractive left-inverse $E_{\mathrm{A}}$ and $R_{\mathbb{C}}$ is furthermore a unital algebra morphism.

We now turn briefly to the $W^{*}$-category.

Definition. A von Neumann bialgebra is a von Neumann algebra $\mathrm{M}$ with coproduct, that is a normal unital ${ }^{*}$-homomorphism $\Delta: M \rightarrow M \bar{\otimes} M$ which is coassociative:

$$
\left(\operatorname{id}_{M} \bar{\otimes} \Delta\right) \circ \Delta=\left(\Delta \bar{\otimes} \operatorname{id}_{M}\right) \circ \Delta .
$$

A counit for $(\mathrm{M}, \Delta)$ is a normal character $\epsilon$ on $\mathrm{M}$ satisfying

$$
\left(\operatorname{id}_{M} \bar{\otimes} \epsilon\right) \circ \Delta=\left(\epsilon \bar{\otimes} \operatorname{id}_{M}\right) \circ \Delta=\operatorname{id}_{M} \text {. }
$$

Convolution in this category is straightforward. Let $\phi_{1} \in C B_{\sigma}\left(\mathrm{M} ; Z_{1}\right)$ and $\phi_{2} \in$ $C B_{\sigma}\left(\mathrm{M} ; Z_{2}\right)$ for $\sigma$-weakly closed concrete operator spaces $Z, Z_{1}$ and $Z_{2}$, then

$$
\phi_{1} \star \phi_{2}:=\left(\phi_{1} \bar{\otimes} \phi_{2}\right) \circ \Delta \in C B_{\sigma}\left(\mathrm{M} ; Z_{1} \bar{\otimes} Z_{2}\right),
$$

so that we may define a map

$$
R_{Z}^{\sigma}: C B_{\sigma}(\mathrm{M} ; Z) \rightarrow C B_{\sigma}(\mathrm{M} ; \mathrm{M} \bar{\otimes} Z), \quad \phi \mapsto \mathrm{id}_{\mathrm{M}} \star \phi=\left(\mathrm{id}_{\mathrm{M}} \bar{\otimes} \phi\right) \circ \Delta .
$$


In particular,

$$
R_{\mathbb{C}}^{\sigma}\left(\varphi_{1} \star \varphi_{2}\right)=R_{\mathbb{C}}^{\sigma} \varphi_{1} \circ R_{\mathbb{C}}^{\sigma} \varphi_{2} \in C B_{\sigma}(\mathrm{M}), \text { for } \varphi_{1}, \varphi_{2} \in \mathrm{M}_{*} .
$$

When $\mathrm{M}$ is counital $R_{Z}^{\sigma}$ has left-inverse

$$
E_{Z}^{\sigma}: C B_{\sigma}(\mathrm{M} ; \mathrm{M} \bar{\otimes} Z) \rightarrow C B_{\sigma}(\mathrm{M} ; Z), \quad \psi \mapsto\left(\epsilon \bar{\otimes} \mathrm{id}_{Z}\right) \circ \psi .
$$

Proposition 2.2. Let $(\mathrm{B}, \Delta)$ be a $C^{*}$-bialgebra. Then $(\overline{\mathrm{B}}, \bar{\Delta})$ is a von Neumann bialgebra. Moreover, if $\epsilon$ is a counit for $\mathrm{B}$ then $\bar{\epsilon}$ is a counit for $\overline{\mathrm{B}}$.

Proof. The map $\bar{\Delta}$ is a normal, unital *-homomorphism and the normal maps

$$
\left(\operatorname{id}_{\overline{\mathrm{B}}} \bar{\otimes} \bar{\Delta}\right) \circ \bar{\Delta} \text { and }\left(\bar{\Delta} \bar{\otimes} \operatorname{id}_{\overline{\mathrm{B}}}\right) \circ \bar{\Delta} \text {. }
$$

agree on $\mathrm{B}$, which is $\sigma$-weakly dense in $\overline{\mathrm{B}}$, and so coincide. In the counital case, $\bar{\epsilon}$ is a normal character on $\overline{\mathrm{B}}$ and the normal maps

$$
\left(\operatorname{id}_{\overline{\mathrm{B}}} \bar{\otimes} \bar{\epsilon}\right) \circ \bar{\Delta},\left(\bar{\epsilon} \bar{\otimes} \mathrm{id}_{\overline{\mathrm{B}}}\right) \circ \bar{\Delta} \text { and } \mathrm{id}_{\overline{\mathrm{B}}}
$$

agree on $\mathrm{B}$ and so coincide.

Naturally, we refer to $(\overline{\mathrm{B}}, \bar{\Delta})$, respectively $(\overline{\mathrm{B}}, \bar{\Delta}, \bar{\epsilon})$ as the universal enveloping von Neumann bialgebra (resp. counital von Neumann bialgebra) of $\mathrm{B}$.

Remark. The two forms of $\mathcal{R}$-map enjoy an easy compatibility: if $\phi \in \operatorname{Lin} C P_{\beta}(\mathrm{B} ; \widetilde{\mathrm{A}})$ for a $C^{*}$-algebra $\mathrm{A}$ then

$$
\overline{R_{\mathrm{A}} \phi}=R_{\overline{\mathrm{A}}}^{\sigma} \bar{\phi},
$$

and similarly for the $E$ maps in the counital case.

From now on we shall denote all maps of the form $R_{Z}^{\sigma}$, respectively $E_{Z}^{\sigma}$, by $\mathcal{R}_{\sigma}$, respectively $\mathcal{E}_{\sigma}$, and similarly abbreviate all maps of the form $R_{\mathrm{A}}$ and $E_{\mathrm{A}}$ to $\mathcal{R}$ and $\mathcal{E}$.

\section{Quantum stochastics}

Fix now, and for the rest of the paper, a complex Hilbert space $\mathrm{k}$ referred to as the noise dimension space. For $c \in \mathrm{k}$ define $\widehat{c}:=\left(\begin{array}{l}1 \\ c\end{array}\right) \in \widehat{\mathrm{k}}$; and for any function $g$ with values in $\mathrm{k}$ let $\widehat{g}$ denote the corresponding function with values in $\widehat{\mathrm{k}}$, defined by $\widehat{g}(s):=\widehat{g(s)}$. Let $\mathcal{F}$ denote the symmetric Fock space over $L^{2}\left(\mathbb{R}_{+} ; \mathrm{k}\right)$, let $\mathbb{S}$ denote the linear span of $\left\{d_{[0, t[}: d \in \mathrm{k}, t \in \mathbb{R}_{+}\right\}$in $L^{2}\left(\mathbb{R}_{+} ; \mathrm{k}\right)$ (for purposes of evaluating, we always take these right-continuous versions) and let $\mathcal{E}$ denote the linear span of $\{\varepsilon(g): g \in \mathbb{S}\}$ in $\mathcal{F}$, where $\varepsilon(g)$ denotes the exponential vector $\left((n !)^{-\frac{1}{2}} g^{\otimes n}\right)_{n>0}$. (There will be no danger of confusion with the inverse of an $\mathcal{R}$-map!) Also define

$$
e_{0}:=\left(\begin{array}{l}
1 \\
0
\end{array}\right) \in \widehat{\mathrm{k}} \text { and } \Delta^{\mathrm{QS}}:=P_{\{0\} \oplus \mathrm{k}}=\left[\begin{array}{ll}
0 & \\
& I_{\mathrm{k}}
\end{array}\right] \in B(\widehat{\mathrm{k}}) .
$$

Quantum stochastic processes, differential equations and cocycles. A detailed summary of the relevant results from QS analysis ([LW $\left.1-4],\left[\mathrm{LS}_{2}\right]\right)$ is given in $\left[\mathrm{LS}_{3}\right]$. We shall therefore be brief here.

For operator spaces $\mathrm{V}$ and $\mathrm{W}$, with $\mathrm{W}$ concrete, $\mathbb{P}(\mathrm{V} \rightarrow \mathrm{W})$ denotes the space of adapted proceses $k=\left(k_{t}\right)_{t \geq 0}$ thus, for $t \in \mathbb{R}_{+}$,

$$
k_{t} \in L\left(\mathcal{E} ; L\left(\mathrm{~V} ; \mathrm{W} \otimes_{\mathrm{M}}|\mathcal{F}\rangle\right)\right) \text {, written } \varepsilon \mapsto k_{t, \varepsilon} .
$$

As in $\left[\mathrm{LS}_{3}\right]$, we abbreviate to $\mathbb{P}_{\star}(\mathrm{V})$ when $\mathrm{W}=\mathbb{C}$. Its associated maps $\kappa_{t}^{f, g}: \mathrm{V} \rightarrow \mathrm{W}$ $\left(f, g \in \mathbb{S}, s, t \in \mathbb{R}_{+}\right)$are defined by

$$
\kappa_{t}^{f^{\prime}, f}(x)=\left(\operatorname{id}_{\mathrm{W}} \otimes_{\mathrm{M}}\left\langle\varepsilon^{\prime}\right|\right) k_{t, \varepsilon}(x), \quad x \in \mathrm{V},
$$


where $\varepsilon=\varepsilon\left(f_{[0, t]}\right)$ and $\varepsilon^{\prime}=\varepsilon\left(f_{[0, t]}^{\prime}\right)$. For us here the pair $(\mathrm{V}, \mathrm{W})$ will be either $(\mathrm{M}, \mathbb{C})$ or $(\mathrm{M}, \mathrm{M})$ for a von Neumann algebra $\mathrm{M}$, or $(\mathrm{A}, \mathbb{C})$ or $(\widetilde{\mathrm{A}}, \mathbb{C})$ for a $C^{*}$-algebra $\mathrm{A}$ with multiplier algebra $\widetilde{A}$. Thus $\mathrm{W} \otimes_{\mathrm{M}}|\mathcal{F}\rangle$ is either $|\mathcal{F}\rangle$ or $\mathrm{M} \bar{\otimes}|\mathcal{F}\rangle$. The process $k$ is weakly initial space bounded if each $\kappa_{t}^{f, g}$ is bounded, and weakly regular if further $\sup \left\{\left\|\kappa_{s}^{f, g}\right\|: s \in[0, T]\right\}<\infty$, for all $T \geq 0$. Here 'column-boundedness' usually obtains: $k_{t, \varepsilon} \in B\left(\mathrm{~V} ; \mathrm{W} \otimes_{\mathrm{M}}|\mathcal{F}\rangle\right)$ or $C B\left(\mathrm{~V} ; \mathrm{W} \otimes_{\mathrm{M}}|\mathcal{F}\rangle\right)$, for each $t \in \mathbb{R}_{+}$and $\varepsilon \in \mathcal{E}$. For von Neumann algebras $\mathrm{M}$ and $\mathrm{N}$, a process $k \in \mathbb{P}(\mathrm{M} \rightarrow \mathrm{N})$ is called normal if each $\kappa_{t}^{f, g}$ is normal. It follows that if $k \in \mathbb{P}(\mathrm{M} \rightarrow \mathrm{N})$ is bounded (meaning that each $k_{t}$ is bounded) and normal then each map $k_{t}$ is normal $\mathrm{M} \rightarrow \mathbf{N} \bar{\otimes} B(\mathcal{F})$. Note that, by Theorem 1.2 , any completely bounded process $l \in \mathbb{P}_{\star}(\mathrm{A})$ on a $C^{*}$-algebra $\mathrm{A}$ is necessarily strict in the sense that each map $l_{t}: \mathrm{A} \rightarrow B(\mathcal{F})$ is strict.

For $\phi \in L\left(\widehat{\mathrm{k}} ; C B\left(\mathrm{~V} ; \mathrm{V} \otimes_{\mathrm{M}}|\widehat{\mathrm{k}}\rangle\right)\right.$ and $\kappa \in C B(\mathrm{~V} ; \mathrm{W})$, where both $\mathrm{V}$ and $\mathrm{W}$ are concrete operator spaces, $k^{\phi, \kappa}$ denotes the unique weakly regular process $k \in \mathbb{P}(\mathrm{V} \rightarrow$ W) satisfying the quantum stochastic differential equation

$$
d k_{t}=k_{t} \cdot d \Lambda_{\phi}(t), \quad k_{0}=\iota_{\mathcal{F}} \circ \kappa,
$$

where $\iota_{\mathcal{F}}$ denotes the ampliation $\mathrm{W} \rightarrow \mathrm{W} \otimes_{\mathrm{M}} B(\mathcal{F}) x \mapsto x \otimes I_{\mathcal{F}}$. The solution is given by

$$
k_{t, \varepsilon}=\sum_{n \geq 0} \Lambda_{t, \varepsilon}^{n} \circ\left(\kappa \circ \phi_{n}\right)
$$

where $\phi_{n}$ is an $n$-fold composition of matrix liftings of $\phi, \Lambda_{t, \varepsilon}^{n}: \mathrm{W} \otimes_{\mathrm{M}}\left|B\left(\widehat{\mathrm{k}}^{\otimes n}\right)\right\rangle \rightarrow$ $\mathrm{W} \otimes_{\mathrm{M}}|\mathcal{F}\rangle$ is the $\varepsilon$-column of the $n$-fold multiple QS integration map, and the sum is norm-convergent in $C B\left(\mathrm{~V} ; \mathrm{W} \otimes_{\mathrm{M}}|\mathcal{F}\rangle\right)$.

When $\mathrm{W}=\mathrm{V}$ and $\kappa=\mathrm{id} \mathrm{V}, k$ is a weak quantum stochastic cocycle on $\mathrm{V}$ (denoted $\left.k^{\phi}\right)$, that is it satisfies $k_{0}=\iota_{\mathcal{F}}$ and for $s, t \in \mathbb{R}_{+}$and $f, g \in \mathbb{S}$,

$$
\kappa_{0}^{f, g}=\mathrm{id} \vee, \quad \kappa_{s+t}^{f, g}=\kappa_{s}^{f, g} \circ \kappa_{t}^{S_{s}^{*} f, S_{s}^{*} g} \quad\left(f, g \in \mathbb{S}, s, t \in \mathbb{R}_{+}\right)
$$

where $\left(S_{t}\right)_{t \geq 0}$ is the isometric semigroup of right-shifts on $L^{2}\left(\mathbb{R}_{+} ; \mathrm{k}\right)$. Let $\left(\sigma_{t}\right)_{t \geq 0}$ denote the induced endomorphism semigroup on $B(\mathcal{F})$, ampliated to $\mathrm{W} \otimes_{\mathrm{M}} B(\mathcal{F})$. Then, when $k$ is a completely bounded process, the cocycle relation simpiflies to

$$
k_{s+t}=k_{s} \cdot \sigma_{s} \circ k_{t},
$$

where the extended composition notation (which we do not need to go into here) is explained in $\left[\mathrm{LS}_{3}\right]$.

\section{Coalgebraic quantum stochastic differential equations}

For this section we fix a $C^{*}$-bialgebra $\mathrm{B}$, which we do not assume to be counital, and consider the coalgebraic quantum stochastic differential equation

$$
d l_{t}=l_{t} \star d \Lambda_{\varphi}(t), \quad l_{0}=\iota_{\mathcal{F}} \circ \eta,
$$

where $\varphi \in S L\left(\widehat{\mathrm{k}}, \widehat{\mathrm{k}} ; \mathrm{B}^{*}\right)$ and $\eta \in \mathrm{B}^{*}$.

Definition. By a form solution of (4.1) is meant a family $\left\{\lambda_{t}^{f, g} \mid f, g \in \mathbb{S}, t \in \mathbb{R}_{+}\right\}$ in $\mathrm{B}^{*}$ satisfying

(i) the map $s \mapsto\left(\lambda_{s}^{f, g} \star \varphi_{\widehat{f}(s), \widehat{g}(s)}\right)(b)$ is locally integrable;

(ii) $\lambda_{t}^{f, g}(b)-e^{\langle f, g\rangle} \eta(b)=\int_{0}^{t} d s\left(\lambda_{s}^{f, g} \star \varphi_{\widehat{f}(s), \widehat{g}(s)}\right)(b)$

for all $f, g \in \mathbb{S}, t \in \mathbb{R}_{+}$and $b \in \mathrm{B}$.

Remarks. Let $f, g \in \mathbb{S}$ and $b \in \mathrm{B}$. By automatic strictness of bounded linear functionals on $\mathrm{B}$, (i) makes sense. By (ii) it follows that $\lambda_{t}^{f, g}(b)$ is continuous in $t$, and so is locally bounded. Therefore, by the Banach-Steinhaus Theorem, $\lambda_{t}^{f, g}$ is locally bounded in $t$ and (ii) therefore implies that (i) refines to 
(i) ${ }^{\prime}$ the map $s \mapsto \lambda_{s}^{f, g}$ is continuous, which in turn implies that (ii) refines to

(ii) ${ }^{\prime} \lambda_{t}^{f, g}-e^{\langle f, g\rangle} \eta=\int_{0}^{t} d s \lambda_{s}^{f, g} \star \varphi_{\widehat{f}(s), \widehat{g}(s)}$,

the integrand being piecewise norm-continuous $\mathbb{R}_{+} \rightarrow \mathrm{B}^{*}$.

The following automatic strictness property is needed to establish uniqueness for form solutions. Recall the strict extension notation $\widetilde{\star}$.

Lemma 4.1. Let $\varphi \in S L\left(\widehat{\mathrm{k}}, \widehat{\mathrm{k}} ; \mathrm{B}^{*}\right)$ and $\eta \in \mathrm{B}^{*}$. Then every form solution $\left\{\lambda_{t}^{f, g} \mid f, g \in\right.$ $\left.\mathbb{S}, t \in \mathbb{R}_{+}\right\}$of $(4.1)$ is strict in the sense that it satisfies

(ii) $\sim \widetilde{\lambda}_{t}^{f, g}-e^{\langle f, g\rangle} \widetilde{\eta}=\int_{0}^{t} d s \lambda_{s}^{f, g} \widetilde{\star} \varphi_{\widehat{f}(s), \widehat{g}(s)}$ for all $f, g \in \mathbb{S}$ and $t \in \mathbb{R}_{+}$, where $\widetilde{\lambda}_{t}^{f, g}:=\left(\lambda_{t}^{f, g}\right) \sim$.

Note that the integrand in (ii) $)^{\sim}$ is piecewise continuous $\mathbb{R}_{+} \rightarrow(\widetilde{B})_{\beta}^{*}$.

Proof. Let $\left\{\lambda_{t}^{f, g} \mid f, g \in \mathbb{S}, t \in \mathbb{R}_{+}\right\}$be a form solution of (4.1) and let $f, g \in \mathbb{S}$ and $t \in \mathbb{R}_{+}$. Define bounded linear functionals

$$
\Phi:=\int_{0}^{t} d s \lambda_{s}^{f, g} \star \varphi_{\widehat{f}(s), \widehat{g}(s)} \text { and } \Psi:=\int_{0}^{t} d s \lambda_{s}^{f, g} \widetilde{\star} \varphi_{\widehat{f}(s), \widehat{g}(s)}
$$

on B and $\widetilde{B}$ respectively. Note that each Riemann approximant $\Psi_{\mathcal{P}}$ of $\Psi$ equals $\left(\Phi_{\mathcal{P}}\right)^{\sim}$ where $\Phi_{\mathcal{P}}$ is the corresponding Riemann approximant of $\Phi$. The extension map $B^{*} \rightarrow \widetilde{B}^{*}$ is (isometric and thus) continuous therefore

$$
\Psi=\lim \Psi_{\mathcal{P}}=\left(\lim \Psi_{\mathcal{P}}\right)^{\sim}=\Phi^{\sim} .
$$

Since $\Phi=\lambda_{t}^{f, g}-e^{\langle f, g\rangle} \eta$ it follows that $\Psi=\left(\lambda_{t}^{f, g}\right)^{\sim}-e^{\langle f, g\rangle} \widetilde{\eta}$. Thus form solution is strict.

With this we have uniqueness as well as existence for form solutions.

Theorem 4.2. Let $\varphi \in S L\left(\widehat{\mathrm{k}}, \widehat{\mathrm{k}} ; \mathrm{B}^{*}\right)$ and $\eta \in \mathrm{B}^{*}$, for a $C^{*}$-bialgebra $\mathrm{B}$. Then the quantum stochastic differential equation (4.1) has a unique form solution.

Proof. For each $c, d \in \mathrm{k}$ let $\left(p_{t}^{c, d}\right)_{t \geq 0}$ denote the norm-continuous one-parameter semigroup generated by $\varphi_{\widehat{c}, \widehat{d}} \in \mathrm{B}^{*}$, in the unitisation of the Banach algebra $\left(\mathrm{B}^{*}, \star\right)$. For $f, g \in \mathbb{S}$ and $t \in \mathbb{R}_{+}$, set

$$
\lambda_{t}^{f, g}:=\eta \star p_{t_{1}-t_{0}}^{c_{0}, d_{0}} \star \cdots \star p_{t_{n+1}-t_{n}}^{c_{n}, d_{n}}
$$

where $t_{0}=0, t_{n+1}=t,\left\{t_{1}<\cdots<t_{n}\right\}$ is the (possibly empty) set of points in ]0, $t$ [ where $f$ or $g$ is discontinuous and $\left(c_{i}, d_{i}\right)=\left(f\left(t_{i}\right), g\left(t_{i}\right)\right)$ for $i=0, \cdots, n$. This defines an element $\lambda_{t}^{f, g}$ of $\mathrm{B}^{*}$. It is easily verified that the resulting family $\left\{\lambda_{t}^{f, g} \mid f, g \in \mathbb{S}, t \in \mathbb{R}_{+}\right\}$is a form solution of (4.1).

Suppose now that $\mu$ is the difference of two form solutions, and let $f, g \in \mathbb{S}$ and $t \in \mathbb{R}_{+}$. Then Lemma 4.1 yields the identity

$$
\left(\mu_{t}^{f, g}\right)^{\sim}=\int_{0}^{t} d s \mu_{s}^{f, g} \approx \varphi_{\widehat{f}(s), \widehat{g}(s)},
$$

which may be iterated. Estimating after repeated iteration (and using the isometry $\widetilde{\mathrm{B}}_{\beta}^{*} \cong \mathrm{B}^{*}$ ) we have

$$
\left\|\mu_{t}^{f, g}\right\| \leq \frac{t^{n}}{n !} \sup _{s \in[0, t]}\left\|\mu_{s}^{f, g}\right\| \max \left\{\left\|\varphi_{\widehat{c}, \widehat{d}}\right\|: c \in \operatorname{Ran} f, d \in \operatorname{Ran} g\right\}^{n}
$$

which tends to 0 as $n \rightarrow \infty$. Thus $\mu=0$, proving uniqueness. 
We now show how stronger forms of solution are obtained when the coefficient of the quantum stochastic differential equation is a bounded mapping rather than just a form. Below the following natural inclusions are invoked:

$$
\begin{aligned}
& B\left(\mathrm{~B} ; B\left(\mathrm{~h} ; \mathrm{h}^{\prime}\right)\right) \cong B\left(\overline{\mathrm{h}^{\prime}}, \mathrm{h} ; \mathrm{B}^{*}\right) \subset S L\left(\mathrm{~h}^{\prime}, \mathrm{h} ; \mathrm{B}^{*}\right), \\
& \varphi \mapsto\left((\zeta, \eta) \mapsto \varphi_{\zeta, \eta}:=\langle\zeta, \varphi(\cdot) \eta\rangle\right),
\end{aligned}
$$

for Hilbert spaces $h$ and $h^{\prime}$. Recall the notation for the solution of a QS differential equation introduced above equation (3.3), and the column notation for processes (3.2).

Theorem 4.3. Let $\varphi \in C B(\mathrm{~B} ; B(\widehat{\mathrm{k}}))$ and $\eta \in \mathrm{B}^{*}$, for a $C^{*}$-bialgebra $\mathrm{B}$. Set

$$
\widetilde{l}^{\varphi, \eta}:=k^{\tilde{\phi}, \tilde{\eta}} \text { and } \bar{l}^{\varphi, \eta}:=k^{\bar{\phi}, \bar{\eta}} \text { where } \phi:=\mathcal{R} \varphi .
$$

Thus $\widetilde{\phi} \in C B_{\beta}(\widetilde{\mathrm{B}} ; \mathrm{B} \widetilde{\otimes} K(\widehat{\mathrm{k}}))$ and $\bar{\phi}=\mathcal{R}_{\sigma} \bar{\varphi} \in C B_{\sigma}(\overline{\mathrm{B}} ; \overline{\mathrm{B}} \bar{\otimes} B(\widehat{\mathrm{k}}))$.

(a) Abbreviating $\bar{l}^{\varphi, \eta}$ to $\bar{l}$ and $\widetilde{l}^{\varphi, \eta}$ to $\widetilde{l}$ we have, for all $\varepsilon \in \mathcal{E}$ and $t \in \mathbb{R}_{+}$,

(i) $\bar{l}_{t, \varepsilon} \in C B_{\sigma}(\overline{\mathrm{B}} ;|\mathcal{F}\rangle)$;

(ii) $\widetilde{l}_{t, \varepsilon}=\left.\bar{l}_{t, \varepsilon}\right|_{\widetilde{\mathrm{B}}}$;

(iii) $\widetilde{l}_{t, \varepsilon} \in C B_{\beta}(\widetilde{\mathrm{B}} ;|\mathcal{F}\rangle)$.

(b) For all $f, g \in \mathbb{S}$ and $t \in \mathbb{R}_{+}$, setting

$$
\bar{\lambda}_{t}^{f, g}:=\omega_{\varepsilon\left(f_{[0, t]}\right), \varepsilon\left(g_{[0, t]}\right)} \circ \bar{l}_{t}^{\varphi, \eta} \text { and } \kappa_{t}^{f, g}:=E^{\varepsilon\left(f_{[0, t]}\right)} k_{t}^{\bar{\phi}}(\cdot) E_{\varepsilon\left(g_{[0, t]}\right)},
$$

(i) $\left\{\left.\bar{\lambda}_{t}^{f, g}\right|_{\mathrm{B}} \mid f, g \in \mathbb{S}, t \in \mathbb{R}_{+}\right\}$is the unique form solution of (4.1);

(ii) $\bar{\lambda}_{t}^{f, g}=\bar{\eta} \circ \kappa_{t}^{f, g}$ and $\mathcal{R}_{\sigma} \bar{\lambda}_{t}^{f, g}=\left(\mathcal{R}_{\sigma} \bar{\eta}\right) \circ \kappa_{t}^{f, g}$.

Proof. Fix $\varepsilon \in \mathcal{E}$ and $t \geq 0$. By linearity we may assume that $\varepsilon=\varepsilon(g)$. Below we adopt the normal extension notation $\alpha \overline{\text { }} \beta:=\overline{\alpha \star \beta}$.

(a) (i) The operator $\bar{l}_{t, \varepsilon}$ is a norm-convergent sum, in $C B(\overline{\mathrm{B}} ;|\mathcal{F}\rangle)$, of terms of the form $\Lambda_{t, \varepsilon}^{n} \circ\left(\eta \bar{\star} \varphi^{\bar{\star} n}\right)\left(n \in \mathbb{Z}_{+}\right)$, and each map $\eta \bar{\star} \varphi^{\bar{\star} n}$ is $\sigma$-weakly continuous. Since $C B_{\sigma}(\overline{\mathrm{B}} ;|\mathcal{F}\rangle)$ is a norm-closed subspace of $C B(\overline{\mathrm{B}} ;|\mathcal{F}\rangle)$ it remains only to show that the bounded operator $\Lambda_{t, \varepsilon}^{n}: B\left(\widehat{\mathrm{k}}^{\otimes n}\right) \rightarrow|\mathcal{F}\rangle$ is $\sigma$-weakly continuous. By the Krein-Smulian Theorem it suffices to prove this on bounded sets. This follows from the following identity for multiple QS integrals:

$$
\left\langle\varepsilon(f), \Lambda_{t}^{n}(A) \varepsilon(g)\right\rangle=\int_{\Delta_{t}^{n}} d \mathbf{s}\left\langle\pi_{\widehat{f}}(\mathbf{s}), A \pi_{\widehat{g}}(\mathbf{s})\right\rangle e^{\langle f, g\rangle}, \quad A \in B\left(\widehat{\mathrm{k}}^{\otimes n}\right),
$$

since the integrand is a step function on $\Delta_{t}^{n}:=\left\{s \in \mathbb{R}^{n}: 0 \leq s_{1} \leq \cdots \leq s_{n} \leq t\right\}$.

(ii) Since $\widetilde{l}_{t, \varepsilon}$ is a norm-convergent sum, in $C B(\widetilde{\mathrm{B}} ;|\mathcal{F}\rangle)$, of terms of the form $\Lambda_{t, \varepsilon}^{n} \circ\left(\eta \widetilde{\star} \varphi^{\widetilde{\star} n}\right)$, this follows from (i) and the identity $\left.\eta \bar{\star} \varphi^{\bar{\star} n}\right|_{\widetilde{\mathrm{B}}}=\eta \widetilde{\star} \varphi^{\widetilde{\star} n}\left(n \in \mathbb{Z}_{+}\right)$.

(iii) This follows from (i) and (ii) since, for any map $\alpha \in C B_{\sigma}(\overline{\mathrm{B}} ;|\mathcal{F}\rangle),\left(\left.\alpha\right|_{\mathrm{B}}\right)^{\sim}=$ $\left(\left.\alpha\right|_{\tilde{\mathrm{B}}}\right)^{\sim}($ see $(1.3))$.

(b) (i) This follows from the identity

$$
\omega_{\varepsilon, \varepsilon^{\prime}} \circ k_{s}^{\bar{\phi}, \bar{\eta}} \circ \bar{\phi}_{\widehat{c}, \widehat{d}}=\omega_{\varepsilon, \varepsilon^{\prime}} \circ k_{s}^{\bar{\phi}, \bar{\eta}} \bar{\star} \bar{\varphi}_{\widehat{c}, \widehat{d}}, \quad \varepsilon, \varepsilon^{\prime} \in \mathcal{E}, c, d \in \mathrm{k}, s \in \mathbb{R}_{+},
$$

where $\bar{\phi}_{\widehat{c}, \widehat{d}}:=\left(\operatorname{id}_{\overline{\mathrm{B}}} \bar{\otimes} \omega_{\widehat{c}, \widehat{d}}\right) \circ \bar{\phi}$.

(ii) The first identity expresses the general relation between $k^{\bar{\phi}, \bar{\eta}}$ and $k^{\bar{\phi}}\left(\left[\mathrm{LS}_{2}\right]\right)$. By (i), it follows from the proof of Theorem 4.2 that $\bar{\lambda}_{t}^{f, g}$ may be written in the form

$$
\bar{\eta} \mp \bar{p}_{t_{1}-t_{0}}^{c_{0}, d_{0}} \mp \cdots \mp \bar{p}_{t_{n+1}-t_{n}}^{c_{n}, d_{n}},
$$


where $\bar{p}_{t}^{c, d}$ denotes the normal extension of $p_{t}^{c, d}$. Thus $\mathcal{R}_{\sigma} \bar{\lambda}_{t}^{f, g}$ equals

$$
\mathcal{R}_{\sigma} \bar{\eta} \circ \bar{P}_{t_{1}-t_{0}}^{c_{0}, d_{0}} \circ \cdots \circ \bar{P}_{t_{n+1}-t_{n}}^{c_{n}, d_{n}}
$$

where $\bar{P}_{t}^{c, d}:=\exp t\left(\bar{\phi}_{\widehat{c}, \widehat{d}}\right)=\mathcal{R}_{\sigma} \bar{p}_{t}^{c, d}$. (ii) therefore now follows from the semigroup representation of the standard QS cocycle $k^{\bar{\phi}}\left(\left[\mathrm{LW}_{2}\right]\right)$.

Notation. Setting $l_{t, \varepsilon}^{\varphi, \eta}=\left.\bar{l}_{t, \varepsilon}^{\varphi, \eta}\right|_{\mathrm{B}}\left(t \in \mathbb{R}_{+}, \varepsilon \in \mathcal{E}\right)$ defines a process $l^{\varphi, \eta} \in \mathbb{P}_{\star}(\mathrm{B})$, which we denote by $l^{\varphi}$ when $\mathrm{B}$ is counital and $\eta=\epsilon$. This extends the notation introduced in $\left[\mathrm{LS}_{3}\right]$ for the unital case.

Remark. In view of the identity

$$
\left(k_{s}^{\bar{\phi}, \bar{\eta}} \circ \phi\right)_{\varepsilon(f), \widehat{f}(s)}=\bar{l}_{s, \varepsilon(f)}^{\varphi, \eta} \bar{\dagger} \bar{f}_{\widehat{f}(s)},
$$

$\bar{l}^{\varphi, \eta}$ satisfies

$$
\bar{l}_{t}=\iota_{\mathcal{F}} \circ \bar{\eta}+\int_{0}^{t} \bar{l}_{s} \mp \bar{\varphi} d \Lambda(s), \quad t \in \mathbb{R}_{+} .
$$

In this sense, $l^{\varphi, \eta}$ is a strong solution of (4.1).

Note that only the coalgebraic structure of B has been used so far, not its algebraic structure.

We end this section by noting some correspondence between convolution processes and associated standard processes. Recall the notation for QSDE solutions introduced above equation (3.4).

Proposition 4.4. Let $l=l^{\varphi}$ and $\bar{l}=\bar{l}^{\varphi}$, where $\varphi \in C B(\mathrm{~B} ; B(\widehat{\mathrm{k}}))$ for a counital $C^{*}$-bialgebra $\mathrm{B}$, and set $k=k^{\bar{\phi}}$ where $\phi:=\mathcal{R} \varphi$. Then

(a) $\bar{l}$ is unital if and only if $k$ is.

(b) $l$ is completely bounded (respectively, completely positive or *-homomorphic) if and only if $k$ is, in which case

$$
k_{t}=\mathcal{R}_{\sigma} \bar{l}_{t}, \quad \bar{l}_{t}=\mathcal{E}_{\sigma} k_{t} \text { and }\left\|l_{t}\right\|_{\mathrm{cb}}=\left\|k_{t}\right\|_{\mathrm{cb}}, \quad t \in \mathbb{R}_{+} .
$$

Proof. In the notations (4.2), Theorem 4.3(b)(ii) implies that,

$$
\bar{\lambda}_{t}^{f, g}=\mathcal{E}_{\sigma} \kappa_{t}^{f, g} \text { and } \kappa_{t}^{f, g}=\mathcal{R}_{\sigma} \bar{\lambda}_{t}^{f, g}, \quad f, g \in \mathbb{S}, t \in \mathbb{R}_{+}
$$

Thus (a) follows from the unitality of the maps $\bar{\epsilon}$ and $\bar{\Delta}$. Moreover, if $k$ is completely bounded then, since

$$
\omega_{\varepsilon, \varepsilon^{\prime}} \circ \bar{l}_{t}^{\varphi, \eta}=\bar{\lambda}_{t}^{f, g}=\mathcal{E}_{\sigma} \kappa_{t}^{f, g}=\omega_{\varepsilon, \varepsilon^{\prime}} \circ \mathcal{E}_{\sigma} k_{t},
$$

where $\varepsilon=\varepsilon\left(f_{[0, t[}\right.$ and $\varepsilon^{\prime}=\varepsilon\left(f_{[0, t}^{\prime}\right.$, for all $f, f^{\prime} \in \mathbb{S}$ and $t \in \mathbb{R}_{+}$, it follows that $l_{t}=\mathcal{E}_{\sigma} k_{t}\left(t \in \mathbb{R}_{+}\right)$, in particular $l$ is completely bounded. Conversely, if $l$ is completely bounded then $\overline{l_{t}}=\bar{l}_{t}\left(t \in \mathbb{R}_{+}\right)$and

$$
\left(\operatorname{id}_{\overline{\mathrm{B}}} \bar{\otimes} \omega_{\varepsilon, \varepsilon^{\prime}}\right) \circ \mathcal{R}_{\sigma} \bar{l}_{t}=\mathcal{R}_{\sigma} \bar{\lambda}_{t}^{f, f^{\prime}}=\kappa_{t}^{f, f^{\prime}}=\left(\operatorname{id}_{\overline{\mathrm{B}}} \bar{\otimes} \omega_{\varepsilon, \varepsilon^{\prime}}\right) \circ k_{t}
$$

for all $f, f^{\prime} \in \mathbb{S}$ and $t \in \mathbb{R}_{+}$, so $k_{t}=\mathcal{R}_{\sigma} \bar{l}_{t}\left(t \in \mathbb{R}_{+}\right)$, therefore $k$ is completely bounded. The rest follows from the fact that $\bar{\Delta}$ and $\bar{\epsilon} \bar{\otimes} \operatorname{id}(\mathcal{F})$ are ${ }^{*}$ homomorphisms. 


\section{Quantum StOchastic CONVOLUTION COCYCleS}

For this section we fix a counital $C^{*}$-bialgebra B.

Definition. A family $\left\{\lambda_{t}^{f, g} \mid f, g \in \mathbb{S}, t \in \mathbb{R}_{+}\right\}$in $\mathrm{B}^{*}$ is a form quantum stochastic convolution cocycle on $\mathrm{B}$ if it satisfies

$$
\lambda_{0}^{f, g}=\epsilon, \quad \lambda_{s+t}^{f, g}=\lambda_{s}^{f, g} \star \lambda_{t}^{S_{s}^{*} f, S_{s}^{*} g}, \quad f, g \in \mathbb{S}, s, t \in \mathbb{R}_{+},
$$

where $\left(S_{t}\right)_{t \geq 0}$ is the isometric shift semigroup on $L^{2}\left(\mathbb{R}_{+} ; \mathrm{k}\right)$.

Note that for such a cocycle

$$
p_{t}^{c, d}:=\lambda_{t}^{c_{[0, t}, d_{[0, t]}}
$$

defines one-parameter semigroups $\left\{p^{c, d}\right\}_{c, d \in \mathrm{k}}$ in the unital Banach algebra $\left(\mathrm{B}^{*}, \star\right)$ which we refer to as the associated convolution semigroups of the cocycle. The cocycle is said to be Markov-regular if each of its associated semigroups is normcontinuous.

Definition. A process $l \in \mathbb{P}_{\star}(\mathrm{B})$ is a (weak) $Q S$ convolution cocycle on $\mathrm{B}$ if its associated family $\left\{\omega_{\varepsilon\left(f_{[0, t]}\right), \varepsilon\left(f_{[0, t]}^{\prime}\right)} \circ l_{t} \mid f, f^{\prime} \in \mathbb{S}, t \in \mathbb{R}_{+}\right\}$is a form QS cocycle on $\mathrm{B}$.

Remarks. (i) Let $l \in \mathbb{P}_{\star}(\mathrm{B})$ be a completely bounded QS convolution cocycle on $\mathrm{B}$. Then $l$ is a QS convolution cocycle in the full sense:

$$
l_{s+t}=l_{s} \star\left(\sigma_{s} \circ l_{t}\right), \quad l_{0}=\iota_{\mathcal{F}} \circ \epsilon, \quad s, t \in \mathbb{R}_{+},
$$

where $\left(\sigma_{s}\right)_{s \geq 0}$ is the injective *-homomorphic semigroup of right shifts on $B(\mathcal{F})$ and the identification

$$
B(\mathcal{F})=B\left(\mathcal{F}_{[0, s[}\right) \bar{\otimes} \sigma_{s}(B(\mathcal{F}))
$$

is invoked.

(ii) It follows from the proof of Theorem 4.2 that, for $\varphi \in S L\left(\widehat{\mathrm{k}}, \widehat{\mathrm{k}} ; \mathrm{B}^{*}\right)$, the unique form solution of the QS differential equation

$$
d l_{t}=l_{t} \star d \Lambda_{\varphi}(t), \quad l_{0}=\iota_{\mathcal{F}} \circ \epsilon,
$$

is a Markov-regular weak QS convolution cocycle on B.

(iii) Form-cocycles may equally be defined on $\widetilde{B}$ and $\bar{B}$ with the requirement of strictness/normality, and $\epsilon$ replaced by $\widetilde{\epsilon}$, respectively $\bar{\epsilon}$. From the correspondence (1.3) it follows that any one of these uniquely determines the others.

Our essential strategy for analysing QS convolution cocycles is to work in the universal enveloping von Neumann bialgebra $\bar{B}$ and, by transferring between convolution and standard QS cocycles using the maps $\mathcal{R}_{\sigma}$ and $\mathcal{E}_{\sigma}$, to apply the theory developed in $\left[\mathrm{LW}_{1-4}\right]$, and $\left[\mathrm{LS}_{2}\right]$.

We first establish a converse to Remark (ii) above.

Proposition 5.1. Let $l$ be a Markov-regular, completely positive, contractive quantum stochastic convolution cocycle on $\mathrm{B}$. Then there is a unique map $\varphi \in C B(\mathrm{~B} ; B(\widehat{\mathrm{k}}))$ such that $l=l^{\varphi}$.

Proof. Set $k:=\left(\mathcal{R}_{\sigma} \bar{l}_{t}\right)_{t \geq 0}$, where $\bar{l}_{t}:=\bar{l}_{t}(t \geq 0)$. Then $k$ is a standard quantum stochastic cocycle on $\overline{\mathrm{B}}$ which is Markov-regular, completely positive, contractive and normal. Therefore, by Theorem 5.10 of $\left[\mathrm{LW}_{2}\right]$ and Theorem 5.3 of $\left[\mathrm{LW}_{1}\right], k$ has a stochastic generator $\bar{\phi} \in C B_{\sigma}(\overline{\mathrm{B}} ; \overline{\mathrm{B}} \bar{\otimes} B(\widehat{\mathrm{k}}))$, moreover for $c, d \in \mathrm{k}$, its associated semigroup $P^{c, d}$ has generator $\left(\operatorname{id}_{\overline{\mathrm{B}}} \bar{\otimes} \omega_{\widehat{c}, \widehat{d}}\right) \circ \bar{\phi}$. Set $\bar{\varphi}:=\mathcal{E}_{\sigma} \bar{\phi} \in C B_{\sigma}(\overline{\mathrm{B}} ; B(\widehat{\mathrm{k}}))$. Since $\bar{l}_{t}=\mathcal{E}_{\sigma} k_{t}$, the associated convolution semigroup $p^{c, d}$ of $\bar{l}$ has generating functional

$$
\bar{\epsilon} \circ\left(\operatorname{id}_{\overline{\mathrm{B}}} \bar{\otimes} \omega_{\widehat{c}, \widehat{d}}\right) \circ \bar{\phi}=\omega_{\widehat{c}, \widehat{d}} \circ \bar{\varphi}
$$


which equals the generating functional of the associated convolution semigroup of the QS convolution cocycle $\bar{l}^{\varphi}$. It follows that $\bar{l}=\bar{l}^{\varphi}$ where $\varphi:=\left.\bar{\varphi}\right|_{\mathrm{B}}$ and so $l=l^{\varphi}$.

We refer to $\varphi$ as the stochastic generator of the QS convolution cocycle $l$. The proof of the next result now proceeds similarly to those of Theorems 5.1 and 6.1 in $\left[\mathrm{LS}_{3}\right]$.

Theorem 5.2. Let l be Markov-regular quantum stochastic convolution cocycle on B. Then the following equivalences hold:

(a) (i) $l$ is completely positive and contractive;

(ii) $l=l^{\varphi}$ for a map $\varphi \in C B(\mathrm{~B} ; B(\widehat{\mathrm{k}}))$ which is expressible in the form $\varphi_{1}-\varphi_{2}$ where $\varphi_{1} \in C P(\mathrm{~B} ; B(\widehat{\mathrm{k}}))$ and $\varphi_{2}=\epsilon(\cdot)\left(\Delta^{\mathrm{QS}}+|\zeta\rangle\left\langle e_{0}|+| e_{0}\right\rangle\langle\zeta|\right)$ for some $\zeta \in \widehat{\mathrm{k}}$, and satisfies $\widetilde{\varphi}(1) \leq 0$.

In this case, the convolution cocycle $l$ is preunital if and only if its stochastic generator $\varphi$ satisfies $\widetilde{\varphi}(1)=0$.

(b) (i) $l$ is completely positive and preunital;

(ii) $l=l^{\varphi}$ for a map $\varphi \in C B(\mathrm{~B} ; B(\widehat{\mathrm{k}}))$ expressible in the form

$$
a \mapsto\left[\begin{array}{c}
\langle\xi| \\
D^{*}
\end{array}\right] \nu(a)\left[\begin{array}{ll}
|\xi\rangle & D
\end{array}\right] \text { for } \nu:=\rho-\iota_{\mathrm{K}} \circ \epsilon,
$$

in which $(\rho, \mathrm{K})$ is a nondegenerate ${ }_{-}^{*}$ representation of $\mathrm{B}\left(\right.$ as $C^{*}$-algebra), $D$ is an isometry in $B(\mathrm{k} ; \mathrm{K})$ and $\xi$ is a vector in $\mathrm{K}$.

(c) (i) $l$ is *-homomorphic;

(ii) $l=l^{\theta}$ where $\theta$ is an $\epsilon$-structure map;

(iii) $l=l^{\theta}$ for a map $\theta$ expressible in the form

$$
a \mapsto\left[\begin{array}{c}
\langle c| \\
I_{\mathrm{k}}
\end{array}\right] \nu(a)\left[|c\rangle \quad I_{\mathrm{k}}\right] \text { where } \nu:=\pi-\iota_{\mathrm{k}} \circ \epsilon,
$$

for a ${ }^{*}$-homomorphism $\pi: \mathrm{B} \rightarrow B(\mathrm{k})$ and vector $c \in \mathrm{k}$.

In this case, the convolution cocycle $l$ is nondegenerate if and only if the *-representation $\pi$ is.

Proof. In case (i) of (a), (b) and (c) we let $\varphi$ be the stochastic generator of $l$, let $\bar{l}=\bar{l}^{\varphi}=\left(\overline{l_{t}}\right)_{t \geq 0}$, and set $k=k^{\bar{\phi}}$ where $\bar{\phi}=\mathcal{R}_{\sigma} \bar{\varphi} \in C B_{\sigma}(\overline{\mathrm{B}} ; \overline{\mathrm{B}} \bar{\otimes} B(\widehat{\mathrm{k}}))$. Thus $k$ is a Markov-regular standard QS cocycle on $\overline{\mathrm{B}}$ and $\bar{\varphi}=\mathcal{E}_{\sigma} \bar{\phi}$.

(a) If (i) holds then $k$ is completely positive and contractive, by Proposition 4.4, and normal. Therefore, by Theorem 5.10 of $\left[\mathrm{LW}_{2}\right]$, there is a map $\Phi \in C P_{\sigma}(\overline{\mathrm{B}} ; \overline{\mathrm{B}} \bar{\otimes} B(\widehat{\mathrm{k}}))$ and operator $Z \in \overline{\mathrm{B}} \bar{\otimes}\langle\widehat{\mathrm{k}}|$ such that

$$
\bar{\phi}(x)=\Phi(x)-\left(x \otimes \Delta^{\mathrm{QS}}+Z^{*}\left(x \otimes\left\langle e_{0}\right|\right)+\left(x \otimes\left|e_{0}\right\rangle\right) Z\right) \quad(x \in \overline{\mathrm{B}})
$$

and $\bar{\phi}(1) \leq 0$. It follows that $\bar{\varphi}(1) \leq 0$ and

$$
\bar{\varphi}=\Psi-\bar{\epsilon}(\cdot)\left(\Delta^{\mathrm{QS}}+|\zeta\rangle\left\langle e_{0}|+| e_{0}\right\rangle\langle\zeta|\right)
$$

where $\Psi=\mathcal{E}_{\sigma} \Phi$ and $\langle\zeta|=\left(\bar{\epsilon} \bar{\otimes} \mathrm{id}_{\langle\widehat{\mathrm{k}}|}\right)(Z)$. Thus (ii) holds with $\psi=\left.\Psi\right|_{\mathrm{B}}$, moreover if $l$ is preunital then $\bar{l}$ is unital and so $k$ is too, therefore $\bar{\phi}(1)=0$ so $\bar{\varphi}(1)=0$ also. Conversely, if (ii) holds then, taking normal extensions,

$$
\bar{\varphi}=\bar{\psi}-\bar{\epsilon}(\cdot)\left(\Delta^{\mathrm{QS}}+|\zeta\rangle\left\langle e_{0}|+| e_{0}\right\rangle\langle\zeta|\right)
$$

and so (5.4) holds with $\Phi=\mathcal{R}_{\sigma} \bar{\psi}$ and $Z=1_{\overline{\mathrm{B}}} \otimes\langle\zeta|$. Therefore, by [LW $\left.\mathrm{LW}_{1}\right]$ Theorem $5.3, k$ is completely positive and contractive and so, by Proposition $4.4, l$ is too. Similarly, if $\bar{\varphi}(1)=0$ then $\bar{\phi}(1)=0$ so $k$ is unital, thus $\bar{l}$ is too, and therefore $l$ is preunital. This proves (a). 
(b) If (i) holds then, choosing $\psi$ and $\zeta$ as in (a), let

$$
\left[\begin{array}{c}
\langle\xi| \\
D^{*}
\end{array}\right] \rho(\cdot)\left[\begin{array}{ll}
|\xi\rangle & D
\end{array}\right]
$$

be a minimal Stinespring decomposition of $\psi$. Thus $(\rho, \mathbf{K})$ is a nondegenerate representation of $\mathrm{B}$ and

$$
\left(\Delta^{\mathrm{QS}}+|\zeta\rangle\left\langle e_{0}|+| e_{0}\right\rangle\langle\zeta|\right)=\widetilde{\psi}(1)=\left[\begin{array}{cc}
\|\xi\|^{2} & \langle\xi| D \\
D^{*}|\xi\rangle & D^{*} D
\end{array}\right]
$$

so $D$ is isometric and (ii) holds. Conversely, suppose that (ii) holds. Then $\bar{\varphi}(1)=0$ and it is easily verified that $\varphi$ has the form given in Part (ii) of (a), with $\zeta=\left(\begin{array}{c}\frac{1}{2}\|\xi\|^{2} \\ D^{*} \xi\end{array}\right)$, therefore (i) holds by (a). This proves (b).

(c) If (i) holds then $k$ is ${ }^{*}$-homomorphic so, by $\left[\mathrm{LW}_{1}\right]$ Proposition $6.3, \bar{\phi}$ is a structure map:

$$
\bar{\phi}\left(x^{*} y\right)=\bar{\phi}(x)^{*} \iota(y)+\iota(x)^{*} \bar{\phi}(y)+\bar{\phi}(x)^{*}\left(1_{\overline{\mathrm{B}}} \otimes \Delta^{\mathrm{QS}}\right) \bar{\phi}(y) \quad(x, y \in \overline{\mathrm{B}}),
$$

where $\iota$ denotes the ampliation map $x \mapsto x \otimes I_{\mathcal{F}}$. Since $\bar{\epsilon} \bar{\otimes} \operatorname{id}_{B(\widehat{\mathrm{k}})}$ is a unital *-homomorphism this implies that

$$
\bar{\varphi}\left(x^{*} y\right)=\bar{\varphi}(x)^{*} \bar{\epsilon}(y)+\bar{\epsilon}(x)^{*} \bar{\varphi}(y)+\bar{\varphi}(x)^{*} \Delta^{\mathrm{QS}} \bar{\varphi}(y) \quad(x, y \in \overline{\mathrm{B}})
$$

and so (ii) holds. Suppose conversely that (ii) holds. By separate $\sigma$-weak continuity of multiplication in $\bar{B}$ it follows that (5.6) holds and a brief calculation confirms the identity

$$
\Omega\left(u^{*} v\right)=\left(\operatorname{id}_{\overline{\mathrm{B}}} \bar{\otimes} \bar{\epsilon}\right)(u)^{*} \Omega(v)+\Omega(u)^{*}\left(\mathrm{id}_{\overline{\mathrm{B}}} \bar{\otimes} \bar{\epsilon}\right)(v)+\Omega(u)^{*}\left(1_{\overline{\mathrm{B}}} \otimes \Delta^{\mathrm{QS}}\right) \Omega(v),
$$

where $\Omega:=\left(\operatorname{id}_{\overline{\mathrm{B}}} \bar{\otimes} \bar{\varphi}\right)$, for simple tensors $u, v \in \overline{\mathrm{B}} \underline{\otimes} \overline{\mathrm{B}}$. Since both sides are separately $\sigma$-weakly continuous the identity is valid for all $u$ and $v$ in $\overline{\mathrm{B}} \bar{\otimes} \overline{\mathrm{B}}$. Substituting in $u=\bar{\Delta} x$ and $v=\bar{\Delta} y$ we see that $\bar{\phi}$ satisfies (5.5). Therefore, by Corollary 4.2 of by $\left[\mathrm{LW}_{4}\right], k$ is $*$-homomorphic thus, by Proposition $4.4, l$ is too and therefore (ii) holds. The equivalence of (ii) and (iii) is the general form of an $\epsilon$-structure map (see (1.12)). In view of (a), the last part is easily seen from the representation (iii). This completes the proof.

Remark. The proper hypothesis for Parts (a) and (b) above is that B be a (multiplier) $C^{*}$-hyperbialgebra, since the multiplicative property of $\Delta$ is not used in their proof. The above result therefore generalises Theorems 5.1 and 6.2 of $\left[\mathrm{LS}_{3}\right]$ to the locally compact category.

\section{Quantum LÉvy PROCESSES ON MUlTiPlier $C^{*}$-BIALGEBRAS}

In this section we extend the definition of weak quantum Lévy process to multiplier $C^{*}$-bialgebras and establish a reconstruction theorem which is analogous to Schürmann's for purely algebraic bialgebras ([Sch]) and extends ours, proved for unital $C^{*}$-bialgebras in $\left[\mathrm{LS}_{3}\right]$.

Throughout this section B denotes a fixed counital $C^{*}$-bialgebra.

Definition. A weak quantum Lévy process on $\mathrm{B}$ over a $C^{*}$-algebra-with-a-state $(\mathrm{A}, \omega)$ is a family $\left(j_{s, t}: \mathrm{B} \rightarrow \widetilde{\mathrm{A}}\right)_{0 \leq s \leq t}$ of nondegenerate ${ }^{*}$-homomorphisms for which the functionals $\lambda_{s, t}:=\omega \circ j_{s, t}$ satisfy the following conditions, for $0 \leq r \leq s \leq t$ :

(i) $\lambda_{r, t}=\lambda_{r, s} \star \lambda_{s, t}$;

(ii) $\lambda_{t, t}=\epsilon$;

(iii) $\lambda_{s, t}=\lambda_{0, t-s}$; 
(iv)

$$
\widetilde{\omega}\left(\prod_{i=1}^{n} j_{s_{i}, t_{i}}\left(x_{i}\right)\right)=\prod_{i=1}^{n} \lambda_{s_{i}, t_{i}}\left(x_{i}\right)
$$

whenever $n \in \mathbb{N}, x_{1}, \ldots, x_{n} \in \mathrm{B}$ and the intervals $\left[s_{1}, t_{1}\left[, \ldots,\left[s_{n}, t_{n}[\right.\right.\right.$ are disjoint;

(v) $\lambda_{0, t} \rightarrow \epsilon$ pointwise as $t \rightarrow 0$.

A weak quantum Lévy process is called Markov-regular if $\lambda_{0, t} \rightarrow \epsilon$ in norm, as $t \rightarrow 0$.

Remarks. In the case of unital $C^{*}$-bialgebras we did not insist that the ${ }^{*}$-algebra A was a $C^{*}$-algebra.

As in the unital case, we refer to the weakly continuous convolution semigroup $\left(\lambda_{t}:=\lambda_{0, t}\right)_{t \geq 0}$ on B as the one-dimensional distribution of the process, and call the process Markov-regular if this is norm-continuous, in which case we refer to the convolution semigroup generator as the generating functional of the process $\left(\left[\mathrm{LS}_{4}\right]\right)$. Moreover, as in the unital case, we call two weak quantum Lévy processes equivalent if their one-dimensional distributions coincide.

The generating functional $\gamma$ of a Markov-regular weak quantum Lévy process, being the generator of a norm-continuous convolution semigroup of states, is real, that is $\gamma=\gamma^{\dagger}$ where $\gamma^{\dagger}(a):=\overline{\gamma\left(a^{*}\right)}$, conditionally positive, that is positive on the ideal $\operatorname{Ker} \epsilon$, and its strict extension satisfies $\widetilde{\gamma}(1)=0$. Note that if $l \in \mathbb{P}_{\star}(\mathrm{B})$ is a QS convolution cocycle on $\mathrm{B}$, with noise dimension space $\mathrm{k}$, which is ${ }^{*}$-homomorphic and preunital then, setting $\mathrm{A}:=K(\mathcal{F}), \omega:=\omega_{\varepsilon(0)}$, and $j_{s, t}:=\sigma_{s} \circ l_{t-s}$ for all $0 \leq s \leq t$, we obtain a weak quantum Lévy process on B, called a Fock space quantum Lévy process, which is Markov-regular if $l$ is. Our goal now is to establish a converse, in other words to extend the reconstruction theorem of $\left[\mathrm{LS}_{3}\right]$ to the nonunital case. We give an elementary self-contained proof, independent of automatic implementability/complete boundedness properties of $\chi$-structure maps. Recall Lemma 1.6.

Theorem 6.1. Let $\gamma \in \mathrm{B}^{*}$ be real, conditionally positive and satisfy $\widetilde{\gamma}(1)=0$. Then there is a (Markov-regular) Fock space quantum Lévy process with generating functional $\gamma$.

Proof. By Theorem 5.2 it suffices to show that there is a Hilbert space $\mathrm{k}$ and an $\epsilon$ structure map $\varphi: B \rightarrow B(\widehat{k})$ of the form $\left[\begin{array}{c}\gamma * \\ * *\end{array}\right]$ satisfying $\widetilde{\varphi}(1)=0$. Set $\widetilde{\gamma}_{0}:=\left.\widetilde{\gamma}\right|_{\text {Ker } \tilde{\epsilon}}$ and let $\psi$ be the map $\mathrm{B} \rightarrow \widetilde{\mathrm{B}}, b \mapsto b-\epsilon(b) 1$. By Theorem 1.1 and Lemma 1.6, $\widetilde{\gamma}$ is real and $\widetilde{\gamma}_{0}$ is positive. Since also $\widetilde{\gamma}(1)=0$,

$$
q:(a, b) \mapsto \gamma\left(a^{*} b\right)-\gamma(a)^{*} \epsilon(b)-\epsilon(a)^{*} \gamma(b)=\widetilde{\gamma}_{0}\left(\psi(a)^{*} \psi(b)\right)
$$

defines a nonnegative sesquilinear form on $\mathrm{B}$. Let $\mathrm{k}$ and $d: \mathrm{B} \rightarrow \mathrm{k}$ be respectively the Hilbert space and induced map obtained by quotienting $B$ by the null space of $q$ and completing, so that

$$
\overline{d(\mathrm{~B})}=\mathrm{k} \text { and }\langle d(a), d(b)\rangle=q(a, b), \quad a, b \in \mathrm{B},
$$

and let $\delta$ be the linear map $\mathrm{B} \rightarrow|\mathrm{k}\rangle, b \mapsto|d(b)\rangle$. Then, by the complete boundedness of $\widetilde{\gamma}$ and $\psi$,

$$
\left\|\delta^{(n)}(A) u\right\|^{2}=\left\langle u,\left(\widetilde{\gamma}_{0}\right)^{(n)}\left(\psi^{(n)}(A)^{*} \psi^{(n)}(A)\right) u\right\rangle \leq\|\widetilde{\gamma}\|_{\mathrm{cb}}\|\psi\|_{\mathrm{cb}}^{2}\|A\|^{2}\|u\|^{2},
$$

for all $n \in \mathbb{N}, A \in M_{n}(\mathrm{~B})$ and $u \in \mathbb{C}^{n}$, so $\delta$ is completely bounded and we have

$$
\delta(a)^{*} \delta(b)=\gamma\left(a^{*} b\right)-\gamma(a)^{*} \epsilon(b)-\epsilon(a)^{*} \gamma(b), \quad a, b \in \mathrm{B} .
$$


Now

$$
\begin{aligned}
\|d(a b)-\epsilon(b) d(a)\|^{2} & =\widetilde{\gamma}_{0}\left(\psi(b)^{*} a^{*} a \psi(b)\right) \\
& \leq\|a\|^{2} \widetilde{\gamma}_{0}\left(\psi(b)^{*} \psi(b)\right)=\|a\|^{2}\|d(b)\|^{2}, \quad a, b \in \mathrm{B},
\end{aligned}
$$

so there are bounded operators $\pi(a)$ on k satisfying

$$
\pi(a) d(b)=d(a b)-\epsilon(b) d(a), \quad a, b \in \mathrm{B} .
$$

Using the density of $d(\mathrm{~B})$ it is straightforward to verify that the map $a \mapsto \pi(a)$ defines a ${ }^{*}$-representation of $\mathrm{B}$ on $\mathrm{k}$. From $(6.3), \delta$ is a $(\pi, \epsilon)$-derivation and so, from (6.2), $\varphi:=\left[\begin{array}{cc}\gamma & \delta^{\dagger} \\ \delta & \pi-\iota_{\mathrm{k}}\end{array}\right]$ defines an $\epsilon$-structure map $\mathrm{B} \rightarrow B(\widehat{\mathrm{k}})$, and therefore it only remains to prove that $\widetilde{\varphi}(1)=0$. Since $\widetilde{\gamma}(1)=0$, this follows from the identities $\delta(a)^{*} \delta(b)=\gamma\left(a^{*} b-\epsilon(a)^{*} b-a^{*} \epsilon(b)\right)$ and $\pi(a) \delta(b)=\delta(a b)-\delta(a) \epsilon(b), \quad a, b \in \mathrm{B}$, and the density of $\bigcup\{\operatorname{Ran} \delta(b): b \in \mathrm{B}\}=d(\mathrm{~B})$ in $\mathrm{k}$.

This has two significant consequences.

Corollary 6.2. Every Markov-regular weak quantum Lévy process is equivalent to a Fock space quantum Lévy process.

The second consequence uses the deeper fact that every $\epsilon$-structure map is implemented (see Theorem 1.5).

Theorem 6.3. Let $\gamma \in \mathrm{B}^{*}$. Then the following are equivalent:

(i) $\gamma$ is the generating functional of a norm-continuous convolution semigroup of states on $\mathrm{B}$;

(ii) $\gamma$ is real, conditionally positive and satisfies $\widetilde{\gamma}(1)=0$;

(iii) There is a nondegenerate representation $(\pi, \mathrm{h})$ of $\mathrm{B}$ and vector $\eta \in \mathrm{h}$ such that $\gamma=\omega_{\eta} \circ\left(\pi-\iota_{\mathrm{h}} \circ \epsilon\right)$.

As stated earlier, the above results mean that all the Poisson-type convolution semigroups of states on B are easily constructed, along with their associated quantum Lévy processes.

In $\left[\mathrm{LS}_{3}\right]$ we also introduced a stronger notion of product system quantum Lévy processes on a unital and counital $C^{*}$-bialgebra $\mathrm{B}$ and established the following two facts: each Fock space quantum Lévy process on $B$ is in particular a product system quantum Lévy process and each product system quantum Lévy process determines in a natural way a weak quantum Lévy process on B with the same finite-dimensional distribution. The definition of a product system quantum Lévy process extends naturally to the nonunital case, with the assumption of unitality - of the *-homomorphisms constituting the process - replaced by nondegeneracy, and the proofs of the above two facts remain valid.

\section{REFERENCES}

[ASW] L. Accardi, M.Schürmann and W. von Waldenfels, Quantum independent increment processes on superalgebras, Math. Z. 198 (1988) no. 4, 451-477.

$[\mathrm{AB}+]$ D. Applebaum, B.V.R. Bhat, J. Kustermans and J.M. Lindsay, "Quantum Independent Increment Processes, Vol. I: From Classical Probability to Quantum Stochastics," eds. U. Franz \& M. Schürmann, Lecture Notes in Mathematics 1865, Springer, Heidelberg 2005.

[BF+] O.E. Barndorff-Nielsen, U. Franz, R. Gohm, B. Kümmerer and S. Thorbjørnsen, "Quantum Independent Increment Processes, Vol. II: Structure of Quantum Lévy Processes, Classical Probability and Physics," eds. U. Franz \&S M. Schürmann, Lecture Notes in Mathematics 1866, Springer, Heidelberg 2006.

[BlM] D.P. Blecher and C. Le Merdy, "Operator Algebras and their Modules - an Operator Space Approach," Oxford University Press, Oxford, 2004.

[ChV] Yu. Chapovsky and L. Vainerman, Compact quantum hypergroups, J. Operator Theory 41 (1999) no. 2, 261-289. 
[EfR] E.G. Effros and Z-J. Ruan, "Operator Spaces," Oxford University Press, 2000.

[Fra] U. Franz, Lévy processes on quantum groups and dual groups, in [BF+].

$\left[\mathrm{Ku}_{1}\right]$ J. Kustermans, One-parameter representations on $C^{*}$-algebras, Preprint, arXiv:functan/9707009.

$\left[\mathrm{Ku}_{2}\right]$ — - Locally compact quantum groups in the universal setting, Internat. J. Math. 12 (2001) no. 3, 289-338.

$\left[\mathrm{Ku}_{3}\right]$ - - Locally compact quantum groups, in $[\mathrm{AB}+]$.

$[\mathrm{KuV}]$ J. Kustermans and S. Vaes, Locally compact quantum groups, Ann. Sci. École Norm. Sup. (4) 33 (2000) no. 6, 837-934.

[Lan] E.C. Lance, "Hilbert $C^{*}$-modules, a toolkit for operator algebraists," LMS Lecture Note Series 210, Cambridge University Press, 1995.

[L] J.M. Lindsay, Quantum stochastic analysis - an introduction, in [AB+].

$\left[\mathrm{LS}_{1}\right]$ J.M. Lindsay and A.G. Skalski, Quantum stochastic convolution cocycles, Ann. Inst. Henri Poincaré (B) 41 (2005), no. 3, 581-604.

$\left[\mathrm{LS}_{2}\right]$ - - On quantum stochastic differential equations, J. Math. Anal. Appl. 330 (2007), no. 2, 1093-1114.

[LS 3$]$ _ - Quantum stochastic convolution cocycles II, Comm. Math. Phys. 280 (2008), no. $3,575-610$.

[LS $]$ - - Convolution semigroups of states, Math. Z. (to appear), Preprint, arXiv:0905.1296.

$\left[\mathrm{LW}_{1}\right] \quad$ J.M. Lindsay and S.J. Wills, Existence, positivity and contractivity for quantum stochastic flows with infinite dimensional noise, Probab. Theory Relat. Fields 116 (2000), 505-543.

$\left[\mathrm{LW}_{2}\right]$ - - Markovian cocycles on operator algebras, adapted to a Fock filtration, J. Funct. Anal. 178 (2000), no. 2, 269-305

$\left[\mathrm{LW}_{3}\right]$ - - Existence of Feller cocycles on a $C^{*}$-algebra, Bull. London Math. Soc. 33 (2001), 613-621.

$\left[\mathrm{LW}_{4}\right]$ - - , Homomorphic Feller cocycles on a $C^{*}$-algebra, J. London Math. Soc. (2) 68 (2003), 255-272.

[Sch] M. Schürmann, "White Noise on Bialgebras," Lecture Notes in Mathematics 1544, Springer, Heidelberg 1993. 